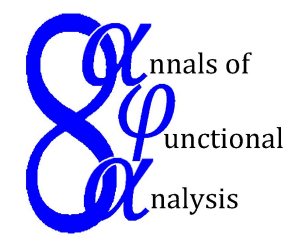

Ann. Funct. Anal. 6 (2015), no. 2, 184-203

http://doi.org/10.15352/afa/06-2-16

ISSN: 2008-8752 (electronic)

http://projecteuclid.org/afa

\title{
A NEW LOOK AT THE CROSSED PRODUCTS OF PRO- $C^{*}$-ALGEBRAS
}

\author{
MARIA JOIŢA
}

Communicated by T. Loring

\begin{abstract}
We give a new definition for the full crossed product, respectively reduced crossed product, of a pro- $C^{*}$-algebra $A\left[\tau_{\Gamma}\right]$ by an action $\alpha$ and, using these new definitions, we investigate some of their properties.
\end{abstract}

\section{INTRODUCTION}

Given a $C^{*}$-algebra $A$ and a continuous action $\alpha$ of a locally compact group $G$ on $A$, we can construct a new $C^{*}$-algebra, called the crossed product of $A$ by $\alpha$, usually denoted by $G \times{ }_{\alpha} A$, and which contains, in some subtle sense, $A$ and $G$. The origin of this construction goes back to Murray and von Neumann and their group measure space construction by which they associated a von Neumann algebra to a countable group acting on a measure space. The analog of this construction for the case of $C^{*}$-algebras is due to Gelfand with co-authors Naimark and Fomin. There is a vast literature on crossed products of $C^{*}$-algebras (see, for example, $[\mathrm{W}]$ ), but the corresponding theory in the context of non-normed topological $*$-algebras has still a long way to go.

Crossed product of pro- $C^{*}$-algebras by inverse limit actions of locally compact groups were considered by Phillips [P2] and Joiţa [J2, J3, J4]. If $A\left[\tau_{\Gamma}\right]$ is a pro$C^{*}$-algebra with topology given by the family of $C^{*}$-seminorms $\Gamma=\left\{p_{\lambda}\right\}_{\lambda \in \Lambda}$, then $A\left[\tau_{\Gamma}\right]$ can be identified with an inverse limit of $C^{*}$-algebras $\lim _{\leftarrow \lambda} A_{\lambda}$ (the ArensMichael decomposition of $A\left[\tau_{\Gamma}\right]$ ), and if $\alpha$ is an inverse limit action of a locally compact group $G$ on $A\left[\tau_{\Gamma}\right]$, then $\alpha_{t}=\lim _{\leftarrow \lambda} \alpha_{t}^{\lambda}$ for all $t \in G$, where for each $\lambda \in \Lambda$, $\alpha^{\lambda}$ is an action of $G$ on the $C^{*}$-algebra $A_{\lambda}$. In [P2], the full (reduced) crossed product of $A\left[\tau_{\Gamma}\right]$ by $\alpha$ is defined as inverse limit of the full (reduced) crossed

Date: Received: Apr. 18, 2014; Accepted: Aug. 3, 2014.

2010 Mathematics Subject Classification. Primary 46L05; Secondary 46H99.

Key words and phrases. pro- $C^{*}$-algebras; crossed products; covariant representations . 
products of $A_{\lambda}$ by $\alpha^{\lambda}, \lambda \in \Lambda$. In particular, for a given inverse limit automorphism $\alpha$ of a pro- $C^{*}$-algebra $A\left[\tau_{\Gamma}\right]$, we can associate to the pair $\left(A\left[\tau_{\Gamma}\right], \alpha\right)$ a pro- $C^{*}$ algebra by the above crossed product construction, but if $\alpha$ is not an inverse limit automorphism, this construction is not possible. In the case of $C^{*}$-algebras, the crossed product of a $C^{*}$-algebra $A$ by an action $\alpha$ is isomorphic to the enveloping $C^{*}$-algebra of the covariance algebra $L^{1}(G, \alpha, A)$. If $\alpha$ is an inverse limit action of $G$ on $A\left[\tau_{\Gamma}\right]$, then the covariance algebra $L^{1}\left(G, \alpha, A\left[\tau_{\Gamma}\right]\right)$ has a structure of locally $m$-convex $*$-algebra with topology given by the family of submultiplicative seminorms $\left\{N_{p_{\lambda}}\right\}_{\lambda \in \Lambda}$, where

$$
N_{p_{\lambda}}(f)=\int_{G} p_{\lambda}(f(g)) d g,
$$

and the enveloping pro- $C^{*}$-algebra of $L^{1}\left(G, \alpha, A\left[\tau_{\Gamma}\right]\right)$ can be identified with the inverse limit of the enveloping $C^{*}$-algebras of the covariance algebras $L^{1}\left(G, \alpha^{\lambda}, A_{\lambda}\right)$. Therefore, the full crossed product of $A\left[\tau_{\Gamma}\right]$ by $\alpha$ is isomorphic to the enveloping pro- $C^{*}$-algebra of the covariance algebra $L^{1}\left(G, \alpha, A\left[\tau_{\Gamma}\right]\right)$. If $\alpha$ is not an inverse limit action, then the covariance algebra has not a structure of locally $m$-convex *-algebra $\left(N_{p_{\lambda}}\right.$ is not a submultiplicative $*$-seminorm). We remark that the above definition of the full crossed product of a pro- $C^{*}$-algebra $A\left[\tau_{\Gamma}\right]$ by an inverse limit action depends of the Arens-Michael decomposition of $A\left[\tau_{\Gamma}\right]$, and so it is not good to define the notion of full crossed product of a pro- $C^{*}$-algebra $A\left[\tau_{\Gamma}\right]$ by an action which is not an inverse limit action. It is well known that the full crossed product of $C^{*}$-algebras is a universal object for nondegenerate covariant representations (see, for example, $[\mathrm{R}]$ ). The full crossed product of pro- $C^{*}$-algebras by inverse limit actions has also the universal property with respect to the nondegenerate covariant representations [J3]. In this paper, we define the full crossed product of a pro- $C^{*}$-algebra $A\left[\tau_{\Gamma}\right]$ by an action $\alpha$ of a locally compact group $G$ as a universal object for nondegenerate covariant representations and we show that the full crossed product of pro- $C^{*}$-algebras exists for strong bounded actions. Strong boundless of the action $\alpha$ is essential to prove the existence of a covariant representation. Unfortunately, if the action $\alpha$ of $G$ on $A\left[\tau_{\Gamma}\right]$ is strongly bounded, then there is another family of $C^{*}$-seminorms on $A\left[\tau_{\Gamma}\right]$ which induces the same topology on $A$, and $\alpha$ is an inverse limit action with respect to this family of $C^{*}$-seminorms.

The organization of this paper is as follows. After preliminaries in Section 2 , we present some examples of group actions on pro- $C^{*}$-algebras in Section 3. In Section 4, we show that for a strong bounded action $\alpha$ of a locally compact group $G$ on a pro- $C^{*}$-algebra $A\left[\tau_{\Gamma}\right]$ there is an injective covariant morphism from $A\left[\tau_{\Gamma}\right]$ to the pro- $C^{*}$-algebra $\mathcal{L}(\mathcal{H})$ for some locally Hilbert space $\mathcal{H}$. In Section 5 , the full pro- $C^{*}$-crossed product of $A\left[\tau_{\Gamma}\right]$ by $\alpha$ is defined to be the pro- $C^{*}$-algebra $G \times_{\alpha} A\left[\tau_{\Gamma}\right]$ generated by the images of $\iota_{A}$ and $\iota_{G}$, where $\left(\iota_{A}, \iota_{G}\right)$ is a universal covariant morphism of $A\left[\tau_{\Gamma}\right]$, in the sense that for any covariant morphism $\left(j_{A}, j_{G}\right)$ from $A\left[\tau_{\Gamma}\right]$ to a pro- $C^{*}$-algebra $B\left[\tau_{\Gamma^{\prime}}\right]$, there is a unique pro$C^{*}$-morphism $\Phi: G \times{ }_{\alpha} A\left[\tau_{\Gamma}\right] \rightarrow B\left[\tau_{\Gamma^{\prime}}\right]$ such that $\Phi \circ \iota_{A}=j_{A}$ and $\Phi \circ \iota_{G}=$ $j_{G}$. For inverse limit actions, this definition coincides with the definition from 
$[\mathrm{P} 2, \mathrm{~J} 2]$. We show that the full pro- $C^{*}$-crossed product of $A\left[\tau_{\Gamma}\right]$ by $\alpha$ exists if $\alpha$ is strongly bounded and it is invariant under the conjugacy of the actions. In Section 6 , the reduced pro- $C^{*}$-crossed product of a pro- $C^{*}$-algebra $A\left[\tau_{\Gamma}\right]$ by a strong bounded action $\alpha$ is defined to be the pro- $C^{*}$-subalgebra of the multiplier algebra $M\left(A\left[\tau_{\Gamma}\right] \otimes_{\min } \mathcal{K}\left(L^{2}(G)\right)\right)$ of the minimal tensor product of $A\left[\tau_{\Gamma}\right]$ and $\mathcal{K}\left(L^{2}(G)\right)$ generated by $\left\{\widetilde{\alpha}(a)\left(1 \otimes \lambda_{G}(f)\right) ; a \in A, f \in C_{c}(G)\right\}$, where $\widetilde{\alpha}$ is the pro- $C^{*}$-morphism from $A\left[\tau_{\Gamma}\right]$ to $M\left(A\left[\tau_{\Gamma}\right] \otimes_{\min } C_{0}(G)\right)$ induced by $\alpha$. We show that, for inverse limit actions, this definition coincides with the definition from $[\mathrm{P} 2, \mathrm{~J} 2]$. Also, we show that the reduced pro- $C^{*}$-crossed product is invariant under the conjugacy of the actions, and if $G$ is amenable, then the full pro$C^{*}$-crossed product of $A\left[\tau_{\Gamma}\right]$ by $\alpha$ is isomorphic to the reduced pro- $C^{*}$-crossed product of $A\left[\tau_{\Gamma}\right]$ by $\alpha$. Section 7 is dedicated the relation between the full pro- $C^{*}$ crossed product and the maximal tensor product of pro- $C^{*}$-algebras, respectively the reduced pro- $C^{*}$-crossed product and the minimal tensor product of pro- $C^{*}$ algebras. We show that there is a property of "associativity" between $\times_{\alpha}$ and $\otimes_{\max }$, respectively $\times_{\alpha, r}$ and $\otimes_{\min }$.

\section{Preliminaries}

A seminorm $p$ on a topological *-algebra $A$ satisfies the $C^{*}$-condition (or is a $C^{*}$-seminorm) if $p\left(a^{*} a\right)=p(a)^{2}$ for all $a \in A$. It is known that such a seminorm must be submultiplicative $(p(a b) \leq p(a) p(b)$ for all $a, b \in A)$ and *-preserving $\left(p\left(a^{*}\right)=p(a)\right.$ for all $\left.a \in A\right)$.

A pro- $C^{*}$-algebra is a complete Hausdorff topological $*$-algebra $A$ whose topology is given by a directed family of $C^{*}$-seminorms $\left\{p_{\lambda}\right\}_{\lambda \in \Lambda}$. Other terms used for pro- $C^{*}$-algebras are: locally $C^{*}$-algebras (A. Inoue, M. Fragoulopoulou, A. Mallios, etc.), $L M C^{*}$-algebras (G. Lassner, K. Schmüdgen), $b^{*}$-algebras (C. Apostol).

Let $A\left[\tau_{\Gamma}\right]$ be a pro- $C^{*}$-algebra with topology given by $\Gamma=\left\{p_{\lambda}\right\}_{\lambda \in \Lambda}$ and let $B\left[\tau_{\Gamma^{\prime}}\right]$ be a pro- $C^{*}$-algebra with topology given by $\Gamma^{\prime}=\left\{q_{\delta}\right\}_{\delta \in \Delta}$. A continuous $*$-morphism $\varphi: A\left[\tau_{\Gamma}\right] \rightarrow B\left[\tau_{\Gamma^{\prime}}\right]$ (that is, $\varphi$ is linear, $\varphi(a b)=\varphi(a) \varphi(b)$ for all $a, b \in A, \varphi\left(a^{*}\right)=\varphi(a)^{*}$ for all $a \in A$ and for each $q_{\delta} \in \Gamma^{\prime}$, there is $p_{\lambda} \in \Gamma$ such that $q_{\delta}(\varphi(a)) \leq p_{\lambda}(a)$ for all $\left.a \in A\right)$ is called a pro- $C^{*}$-morphism. Two pro$C^{*}$-algebras $A\left[\tau_{\Gamma}\right]$ and $B\left[\tau_{\Gamma^{\prime}}\right]$ are isomorphic if there is a pro- $C^{*}$-isomorphism $\varphi: A\left[\tau_{\Gamma}\right] \rightarrow B\left[\tau_{\Gamma^{\prime}}\right]$ (that is, $\varphi$ is invertible, $\varphi$ and $\varphi^{-1}$ are pro- $C^{*}$-morphisms).

If $\left\{A_{\lambda} ; \pi_{\lambda \mu}\right\}_{\lambda \geq \mu, \lambda, \mu \in \Lambda}$ is an inverse system of $C^{*}$-algebras, then $\lim _{\leftarrow \lambda} A_{\lambda}$ with topology given by the family of $C^{*}$-seminorms $\left\{p_{\lambda}\right\}_{\lambda \in \Lambda}$, with $p_{\lambda}\left(\left(a_{\mu}\right)_{\mu \in \Lambda}\right)=$ $\left\|a_{\lambda}\right\|_{A_{\lambda}}$ for all $\lambda \in \Lambda$, is a pro- $C^{*}$-algebra.

Let $A\left[\tau_{\Gamma}\right]$ be a pro- $C^{*}$-algebra with topology given by $\Gamma=\left\{p_{\lambda}\right\}_{\lambda \in \Lambda}$. For $\lambda \in \Lambda$, ker $p_{\lambda}$ is a closed $*$-bilateral ideal and $A_{\lambda}=A / \operatorname{ker} p_{\lambda}$ is a $C^{*}$-algebra in the $C^{*}$ norm $\|\cdot\|_{p_{\lambda}}$ induced by $p_{\lambda}$ (that is, $\|a\|_{p_{\lambda}}=p_{\lambda}(a)$, for all $a \in A$ ). The canonical map from $A$ to $A_{\lambda}$ is denoted by $\pi_{\lambda}^{A}, \pi_{\lambda}^{A}(a)=a+\operatorname{ker} p_{\lambda}$ for all $a \in A$. For $\lambda, \mu \in \Lambda$ with $\mu \leq \lambda$ there is a unique surjective $C^{*}$-morphism $\pi_{\lambda \mu}^{A}: A_{\lambda} \rightarrow A_{\mu}$ such that $\pi_{\lambda \mu}^{A}\left(a+\operatorname{ker} p_{\lambda}\right)=a+\operatorname{ker} p_{\mu}$, and then $\left\{A_{\lambda} ; \pi_{\lambda \mu}^{A}\right\}_{\lambda, \mu \in \Lambda}$ is an inverse system of 
$C^{*}$-algebras. Moreover, pro- $C^{*}$-algebras $A\left[\tau_{\Gamma}\right]$ and $\lim _{\leftarrow \lambda} A_{\lambda}$ are isomorphic (the Arens-Michael decomposition of $\left.A\left[\tau_{\Gamma}\right]\right)$.

Let $\left\{\left(\mathcal{H}_{\lambda},\langle\cdot, \cdot\rangle_{\lambda}\right)\right\}_{\lambda \in \Lambda}$ be a family of Hilbert spaces such that $\mathcal{H}_{\mu} \subseteq \mathcal{H}_{\lambda}$ and $\left.\langle\cdot, \cdot\rangle_{\lambda}\right|_{\mathcal{H}_{\mu}}=\langle\cdot, \cdot\rangle_{\mu}$ for all $\lambda, \mu \in \Lambda$ with $\mu \leq \lambda . \mathcal{H}=\lim _{\lambda \rightarrow} \mathcal{H}_{\lambda}$ with inductive limit topology is called a locally Hilbert space.

Let $\mathcal{L}(\mathcal{H})=\left\{T: \mathcal{H} \rightarrow \mathcal{H} ; T_{\lambda}=\left.T\right|_{\mathcal{H}_{\lambda}} \in L\left(\mathcal{H}_{\lambda}\right)\right.$ and $P_{\lambda \mu} T_{\lambda}=T_{\lambda} P_{\lambda \mu}$ for all $\lambda, \mu \in \Lambda$ with $\mu \leq \lambda\}$, where $P_{\lambda \mu}$ is the projection of $\mathcal{H}_{\lambda}$ on $\mathcal{H}_{\mu}$. Clearly, $\mathcal{L}(\mathcal{H})$ is an algebra in an obvious way, and $T \rightarrow T^{*}$ with $\left.T^{*}\right|_{\mathcal{H}_{\lambda}}=\left(T_{\lambda}\right)^{*}$ for all $\lambda \in \Lambda$ is an involution.

For each $\lambda \in \Lambda$, the map $p_{\lambda, \mathcal{L}(\mathcal{H})}: \mathcal{L}(\mathcal{H}) \rightarrow[0, \infty)$ given by $p_{\lambda, \mathcal{L}(\mathcal{H})}(T)=$ $\left\|\left.T\right|_{\mathcal{H}_{\lambda}}\right\|_{L\left(\mathcal{H}_{\lambda}\right)}$ is a $C^{*}$-seminorm on $\mathcal{L}(\mathcal{H})$, and with topology given by the family of $C^{*}$-seminorms $\left\{p_{\lambda, \mathcal{L}(\mathcal{H})}\right\}_{\lambda \in \Lambda}, \mathcal{L}(\mathcal{H})$ becomes a pro- $C^{*}$-algebra.

Since $\mathcal{L}(\mathcal{H})$ is a pro- $C^{*}$-algebra, it has an Arens-Michael decomposition, given by the $C^{*}$-algebras $\mathcal{L}(\mathcal{H})_{\lambda}=\mathcal{L}(\mathcal{H}) / \operatorname{ker} p_{\lambda, \mathcal{L}(\mathcal{H})}, \lambda \in \Lambda$. Moreover, for each $\lambda \in$ $\Lambda$, the map $\varphi_{\lambda}: \mathcal{L}(\mathcal{H})_{\lambda} \rightarrow L\left(\mathcal{H}_{\lambda}\right)$ given by $\varphi_{\lambda}\left(T+\operatorname{ker} p_{\lambda, \mathcal{L}(\mathcal{H})}\right)=\left.T\right|_{\mathcal{H}_{\lambda}}$ is an isometric *-morphism. The canonical maps from $\mathcal{L}(\mathcal{H})$ to $\mathcal{L}(\mathcal{H})_{\lambda}, \lambda \in \Lambda$ are denoted by $\pi_{\lambda}^{\mathcal{H}}, \lambda \in \Lambda$, and $\pi_{\lambda}^{\mathcal{H}}(T)=\left.T\right|_{\mathcal{H}_{\lambda}}$. For a given pro- $C^{*}$-algebra $A\left[\tau_{\Gamma}\right]$ there is a locally Hilbert space $\mathcal{H}$ such that $A\left[\tau_{\Gamma}\right]$ is isomorphic to a pro- $C^{*}$ subalgebra of $\mathcal{L}(\mathcal{H})$ (see [I, Theorem 5.1]).

A multiplier of $A\left[\tau_{\Gamma}\right]$ is a pair $(l, r)$ of linear maps $l, r: A\left[\tau_{\Gamma}\right] \rightarrow A\left[\tau_{\Gamma}\right]$ such that are respectively left and right $A$-module homomorphisms and $r(a) b=a l(b)$ for all $a, b \in A$. The set $M\left(A\left[\tau_{\Gamma}\right]\right)$ of all multipliers of $A\left[\tau_{\Gamma}\right]$ is a pro- $C^{*}$-algebra with multiplication given by $\left(l_{1}, r_{1}\right)\left(l_{2}, r_{2}\right)=\left(l_{1} l_{2}, r_{2} r_{1}\right)$, the involution given by $(l, r)^{*}=\left(r^{*}, l^{*}\right)$, where $r^{*}(a)=r\left(a^{*}\right)^{*}$ and $l^{*}(a)=l\left(a^{*}\right)^{*}$ for all $a \in A$, and the topology given by the family of $C^{*}$-seminorms $\left\{p_{\lambda, M\left(A\left[\tau_{\Gamma}\right]\right)}\right\}_{\lambda \in \Lambda}$, where $p_{\lambda, M\left(A\left[\tau_{\Gamma}\right]\right)}(l, r)=\sup \left\{p_{\lambda}(l(a)) ; p_{\lambda}(a) \leq 1\right\}$. Moreover, for each $p_{\lambda} \in \Gamma$, the $C^{*}$-algebras $\left(M\left(A\left[\tau_{\Gamma}\right]\right)\right)_{\lambda}$ and $M\left(A_{\lambda}\right)$ are isomorphic. The strict topology on $M\left(A\left[\tau_{\Gamma}\right]\right)$ is given by the family of seminorms $\left\{p_{\lambda, a}\right\}_{(\lambda, a) \in \Lambda \times A}$, where $p_{\lambda, a}(l, r)=$ $p_{\lambda}(l(a))+p_{\lambda}(r(a)), M\left(A\left[\tau_{\Gamma}\right]\right)$ is complete with respect to the strict topology and $A\left[\tau_{\Gamma}\right]$ is dense in $M\left(A\left[\tau_{\Gamma}\right]\right)$ (see [P1] and [J1, Proposition 3.4]).

A pro- $C^{*}$-morphism $\varphi: A\left[\tau_{\Gamma}\right] \rightarrow M\left(B\left[\tau_{\Gamma^{\prime}}\right]\right)$ is nondegenerate if $[\varphi(A) B]=$ $B\left[\tau_{\Gamma^{\prime}}\right]$, where $[\varphi(A) B]$ denotes the closed subspace of $B\left[\tau_{\Gamma^{\prime}}\right]$ generated by $\{\varphi(a) b$; $a \in A, b \in B\}$. A nondegenerate pro- $C^{*}$-morphism $\varphi: A\left[\tau_{\Gamma}\right] \rightarrow M\left(B\left[\tau_{\Gamma^{\prime}}\right]\right)$ extends to a unique pro- $C^{*}$-morphism $\bar{\varphi}: M\left(A\left[\tau_{\Gamma}\right]\right) \rightarrow M\left(B\left[\tau_{\Gamma^{\prime}}\right]\right)$.

\section{Group aCtions on PRO- $C^{*}$-ALGEBras}

Throughout this paper, $A\left[\tau_{\Gamma}\right]$ is a pro- $C^{*}$-algebra with topology given by the family of $C^{*}$-seminorms $\Gamma=\left\{p_{\lambda}\right\}_{\lambda \in \Lambda}$ and $G$ is a locally compact group.

Definition 3.1. (1) An action of $G$ on $A\left[\tau_{\Gamma}\right]$ is a group morphism $\alpha$ from $G$ to $\operatorname{Aut}\left(A\left[\tau_{\Gamma}\right]\right)$, the group of all automorphisms of $A\left[\tau_{\Gamma}\right]$, such that the map $t \mapsto \alpha_{t}(a)$ from $G$ to $A\left[\tau_{\Gamma}\right]$ is continuous for each $a \in A$.

(2) An action $\alpha$ of $G$ on $A\left[\tau_{\Gamma}\right]$ is strongly bounded, if for each $\lambda \in \Lambda$ there is $\mu \in \Lambda$ such that

$$
p_{\lambda}\left(\alpha_{t}(a)\right) \leq p_{\mu}(a)
$$


for all $t \in G$ and for all $a \in A$.

(3) An action $\alpha$ is an inverse limit action, if $p_{\lambda}\left(\alpha_{t}(a)\right)=p_{\lambda}(a)$ for all $a \in A$, for all $t \in G$ and for all $\lambda \in \Lambda$.

Remark 3.2. (1) If $\alpha$ is an inverse limit action of $G$ on $A\left[\tau_{\Gamma}\right]$, then for each $\lambda \in \Lambda$, there is an action $\alpha^{\lambda}$ of $G$ on $A_{\lambda}$ such that $\alpha_{t}^{\lambda} \circ \pi_{\lambda}^{A}=\pi_{\lambda}^{A} \circ \alpha_{t}$ for all $t \in G$, and $\alpha_{t}=\lim _{\llcorner\lambda} \alpha_{t}^{\lambda}$ for all $t \in G$.

(2) Any inverse limit action of $G$ on $A\left[\tau_{\Gamma}\right]$ is strongly bounded.

(3) If $A$ is a $C^{*}$-algebra, then any action of $G$ on $A$ is strongly bounded.

(4) If $G$ is a compact group, then any action of $G$ on $A\left[\tau_{\Gamma}\right]$ is strongly bounded.

Let $X$ be a compactly countably generated Hausdorff topological space (that is, $X$ is a direct limit of a countable family $\left\{K_{n}\right\}_{n}$ of compact spaces). The $*-$ algebra $C(X)$ of all continuous complex valued functions on $X$ is a pro- $C^{*}$-algebra with topology given by the family of $C^{*}$-seminorms $\left\{p_{K_{n}}\right\}_{n}$, where $p_{K_{n}}(f)=$ $\sup \left\{|f(x)| ; x \in K_{n}\right\}$.

Example 3.3. Let $(G, X)$ be a transformation group (that is, there is a continuous map $(t, x) \mapsto t \cdot x$ from $G \times X$ to $X$ such that $e \cdot x=x$ and $s \cdot(t \cdot x)=(s t) \cdot x$ for all $s, t \in G$ and for all $x \in X$ ) with $X=\lim _{n \rightarrow} K_{n}$ a compactly countably generated Hausdorff topological space. Then there is an action $\alpha$ of $G$ on the pro- $C^{*}$-algebra $C(X)$, given by

$$
\alpha_{t}(f)(x)=f\left(t^{-1} \cdot x\right) .
$$

If for any positive integer $n$, there is a positive integer $m$ such that $G \cdot K_{n} \subseteq K_{m}$, the action $\alpha$ is strongly bounded, since for each $n$, there is $m$ such that

$$
p_{K_{n}}\left(\alpha_{t}(f)\right)=\sup \left\{\left|f\left(t^{-1} \cdot x\right)\right| ; x \in K_{n}\right\} \leq \sup \left\{|f(y)| ; y \in K_{m}\right\}=p_{K_{m}}(f)
$$

for all $f \in C(X)$ and for all $t \in G$. If $G \cdot K_{n}=K_{n}$ for all $n$, then $\alpha$ is an inverse limit action. Take, for instance, $\mathbb{R}=\lim _{n \rightarrow}[-n, n]$. Suppose that $\mathbb{Z}_{2}$ actions on $\mathbb{R}$ by $\widehat{0} \cdot x=x$ and $\widehat{1} \cdot x=2-x$ for all $x \in \mathbb{R}$. Then $\left(\mathbb{Z}_{2}, \mathbb{R}\right)$ is a transformation group such that for each positive integer $n, \mathbb{Z}_{2} \cdot[-n, n] \subseteq[-n-2, n+2]$.

Example 3.4. Let $X=\lim K_{n}$ be a compactly countably generated Hausdorff topological space and $h: \stackrel{n \rightarrow}{X} \rightarrow X$ a homeomorphism with the property that for each positive integer $n$, there is a positive integer $m$ such that $h^{k}\left(K_{n}\right) \subseteq K_{m}$ for all integers $k$. Then the map $n \mapsto \alpha_{n}$ from $\mathbb{Z}$ to $\operatorname{Aut}(C(X))$, where $\alpha_{n}(f)=f \circ h^{n}$, is a strong bounded action of $\mathbb{Z}$ to $C(X)$. If $h\left(K_{n}\right)=K_{n}$ for all $n$, then $\alpha$ is an inverse limit action. Take, for instance, $\mathbb{R}=\lim _{n \rightarrow}[-n, n]$. The map $h: \mathbb{R} \rightarrow \mathbb{R}$ defined by $h(x)=1-x$ is a homeomorphism such that for each positive integer $n, h^{k}([-n, n]) \subseteq[-n-1, n+1]$ for all integers $k$.

Example 3.5. The *-algebra $C[0,1]$ equipped with the topology 'cc' of uniform convergence on countable compact subsets is a pro- $C^{*}$-algebra denoted by $C_{c c}[0,1]$ (see, for example, [F, p. 104]). The action of $\mathbb{Z}_{2}$ on $C_{c c}[0,1]$ given by $\alpha_{\widehat{0}}=\operatorname{id}_{C_{c c}[0,1]}$ and $\alpha_{\hat{1}}(f)(x)=f(1-x)$ for all $f \in C_{c c}[0,1]$ and for all $x \in[0,1]$ is strongly bounded. 
Remark 3.6. (1) Let $\alpha$ be a strong bounded action of $G$ on $A\left[\tau_{\Gamma}\right]$. Then, for each $\lambda \in \Lambda$, the map $p^{\lambda}: A \rightarrow[0, \infty)$ given by

$$
p^{\lambda}(a)=\sup \left\{p_{\lambda}\left(\alpha_{t}(a)\right) ; t \in G\right\}
$$

is a continuous $C^{*}$-seminorm on $A\left[\tau_{\Gamma}\right]$. Let $\Gamma^{G}=\left\{p^{\lambda}\right\}_{\lambda \in \Lambda}$. Since, for each $\lambda \in \Lambda$, there is $\mu \in \Lambda$ such that

$$
p_{\lambda} \leq p^{\lambda} \leq p_{\mu}
$$

$\Gamma^{G}$ defines on $A$ a structure of pro- $C^{*}$-algebra, and moreover, the pro- $C^{*}$ algebras $A\left[\tau_{\Gamma}\right]$ and $A\left[\tau_{\Gamma}^{G}\right]$ are isomorphic.

(2) If the action $\alpha$ of $G$ on $A\left[\tau_{\Gamma}\right]$ is strongly bounded, then $\alpha$ is an inverse limit action of $G$ on $A\left[\tau_{\Gamma}^{G}\right]$.

\section{Covariant Representations}

Definition 4.1. A pro- $C^{*}$-dynamical system is a triple $\left(G, \alpha, A\left[\tau_{\Gamma}\right]\right)$, where $G$ is a locally compact group, $A\left[\tau_{\Gamma}\right]$ is a pro- $C^{*}$-algebra and $\alpha$ is an action of $G$ on $A\left[\tau_{\Gamma}\right]$.

$A$ representation of a pro- $C^{*}$-algebra $A\left[\tau_{\Gamma}\right]$ on a Hilbert space $\mathcal{H}$ is a continuous *-morphism $\varphi: A\left[\tau_{\Gamma}\right] \rightarrow L(\mathcal{H})$. A representation $(\varphi, \mathcal{H})$ of $A\left[\tau_{\Gamma}\right]$ is nondegenerate if $[\varphi(A) \mathcal{H}]=\mathcal{H}$

Definition 4.2. A covariant representation of $\left(G, \alpha, A\left[\tau_{\Gamma}\right]\right)$ on a Hilbert space $\mathcal{H}$ is a triple $(\varphi, u, \mathcal{H})$ consisting of a representation $(\varphi, \mathcal{H})$ of $A\left[\tau_{\Gamma}\right]$ on $\mathcal{H}$ and a unitary *-representation $(u, \mathcal{H})$ of $G$ on $\mathcal{H}$ such that

$$
\varphi\left(\alpha_{t}(a)\right)=u_{t} \varphi(a) u_{t}^{*}
$$

for all $a \in A$ and for all $t \in G$. A covariant representation $(\varphi, u, \mathcal{H})$ is nondegenerate if $(\varphi, \mathcal{H})$ is nondegenerate.

Two representations $(\varphi, u, \mathcal{H})$ and $(\psi, v, \mathcal{K})$ of $\left(G, \alpha, A\left[\tau_{\Gamma}\right]\right)$ are unitarily equivalent if there is a unitary operator $U: \mathcal{H} \rightarrow \mathcal{K}$ such that $U \varphi(a)=\psi(a) U$ for all $a \in A$ and $U u_{t}=v_{t} U$ for all $t \in G$.

For each $p_{\lambda} \in \Gamma$, we denote by $\mathcal{R}_{\lambda}\left(G, \alpha, A\left[\tau_{\Gamma}\right]\right)$ the collection of all unitary equivalence classes of nondegenerate covariant representations $(\varphi, u, \mathcal{H})$ of $\left(G, \alpha, A\left[\tau_{\Gamma}\right]\right)$ with the property that $\|\varphi(a)\| \leq p_{\lambda}(a)$ for all $a \in A$. Clearly,

$$
\bigcup_{\lambda} \mathcal{R}_{\lambda}\left(G, \alpha, A\left[\tau_{\Gamma}\right]\right)=\mathcal{R}\left(G, \alpha, A\left[\tau_{\Gamma}\right]\right)
$$

where $\mathcal{R}\left(G, \alpha, A\left[\tau_{\Gamma}\right]\right)$ denotes the collection of all unitary equivalence classes of nondegenerate covariant representations of $\left(G, \alpha, A\left[\tau_{\Gamma}\right]\right)$.

Remark 4.3. If $\alpha$ is an inverse limit action, then the map

$$
\left(\varphi_{\lambda}, u, \mathcal{H}\right) \rightarrow\left(\varphi_{\lambda} \circ \pi_{\lambda}^{A}, u, \mathcal{H}\right)
$$

is a bijection between $\mathcal{R}\left(G, \alpha^{\lambda}, A_{\lambda}\right)$ and $\mathcal{R}_{\lambda}\left(G, \alpha, A\left[\tau_{\Gamma}\right]\right)$ (see, [J2]). 
By [J2], if $\alpha$ is an inverse limit action, then $\mathcal{R}\left(G, \alpha, A\left[\tau_{\Gamma}\right]\right)$ is non empty. From this result and Remark 3.6, we conclude that if $\alpha$ is strongly bounded, then $\mathcal{R}\left(G, \alpha, A\left[\tau_{\Gamma}\right]\right)$ is non empty too. In the following proposition we give another proof for this result.

Proposition 4.4. Let $\left(G, \alpha, A\left[\tau_{\Gamma}\right]\right)$ be a pro- $C^{*}$-dynamical system such that $\alpha$ is strongly bounded. Then there is a covariant representation of $\left(G, \alpha, A\left[\tau_{\Gamma}\right]\right)$.

Proof. Let $(\varphi, \mathcal{H})$ be a representation of $A\left[\tau_{\Gamma}\right]$. Then there is $\lambda \in \Lambda$ such that $\|\varphi(a)\| \leq p_{\lambda}(a)$ for all $a \in A$. Let $a \in A$ and $\xi \in L^{2}(G, \mathcal{H})$. Since, there is $p_{\mu} \in \Gamma$ such that

$$
\begin{aligned}
\int_{G}\left\|\varphi\left(\alpha_{s^{-1}}(a)\right)(\xi(s))\right\|^{2} d s & \leq \int_{G}\left\|\varphi\left(\alpha_{s^{-1}}(a)\right)\right\|^{2}\|\xi(s)\|^{2} d s \\
& \leq \int_{G} p_{\lambda}\left(\alpha_{s^{-1}}(a)\right)^{2}\|\xi(s)\|^{2} d s \leq p_{\mu}(a)^{2}\|\xi\|^{2}
\end{aligned}
$$

the map $s \mapsto \varphi\left(\alpha_{s^{-1}}(a)\right)(\xi(s))$ defines an element in $L^{2}(G, \mathcal{H})$. Therefore, there is $\widetilde{\varphi}(a) \in L\left(L^{2}(G, \mathcal{H})\right)$ such that

$$
\widetilde{\varphi}(a)(\xi)(s)=\varphi\left(\alpha_{s^{-1}}(a)\right)(\xi(s)) .
$$

In this way, we obtain a map $\widetilde{\varphi}: A \rightarrow L\left(L^{2}(G, \mathcal{H})\right)$. Moreover, $\widetilde{\varphi}$ is a continuous *-morphism, and then $\left(\widetilde{\varphi}, L^{2}(G, \mathcal{H})\right)$ is a representation of $A\left[\tau_{\Gamma}\right]$.

Let $\left(\lambda_{G}^{\mathcal{H}}, L^{2}(G, \mathcal{H})\right)$ be the unitary *-representation of $G$ on $L^{2}(G, \mathcal{H})$ given by $\left(\lambda_{G}^{\mathcal{H}}\right)_{t}(\xi)(s)=\xi\left(t^{-1} s\right)$. It is easy to verify that $\left(\widetilde{\varphi}, \lambda_{G}^{\mathcal{H}}, L^{2}(G, \mathcal{H})\right)$ is a covariant representation of $\left(G, \alpha, A\left[\tau_{\Gamma}\right]\right)$.

Remark 4.5. Let $\left(G, \alpha, A\left[\tau_{\Gamma}\right]\right)$ be a pro- $C^{*}$-dynamical system. Suppose that $\alpha$ is strongly bounded. Then, for each representation $(\varphi, \mathcal{H})$ of $A\left[\tau_{\Gamma}\right], \operatorname{ker} \widetilde{\varphi} \subseteq \operatorname{ker} \varphi$. Indeed, if $\widetilde{\varphi}(a)=0$, then $\varphi\left(\alpha_{s}(a)\right)(\xi(s))=0$ for all $s \in G$ and for all $\xi \in$ $L^{2}(G, \mathcal{H})$, whence $\varphi(a)(\xi(e))=0$ for all $\xi \in L^{2}(G, \mathcal{H})$ and so $\varphi(a)=0$.

Definition 4.6. A covariant pro- $C^{*}$-morphism from $\left(G, \alpha, A\left[\tau_{\Gamma}\right]\right)$ to a pro- $C^{*}-$ algebra $B\left[\tau_{\Gamma^{\prime}}\right]$ is a pair $(\varphi, u)$ consisting of a pro- $C^{*}$-morphism $\varphi: A\left[\tau_{\Gamma}\right] \rightarrow$ $M\left(B\left[\tau_{\Gamma^{\prime}}\right]\right)$ and a strict continuous group morphism $u: G \rightarrow \mathcal{U}\left(M\left(B\left[\tau_{\Gamma^{\prime}}\right]\right)\right)$, the group of all unitaries of $M\left(B\left[\tau_{\Gamma^{\prime}}\right]\right)$, such that

$$
\varphi\left(\alpha_{t}(a)\right)=u_{t} \varphi(a) u_{t}^{*}
$$

for all $t \in G$ and for all $a \in A$. A covariant pro- $C^{*}$-morphism $(\varphi, u)$ from $\left(G, \alpha, A\left[\tau_{\Gamma}\right]\right)$ to $B\left[\tau_{\Gamma^{\prime}}\right]$ is nondegenerate if $[\varphi(A) B]=B\left[\tau_{\Gamma^{\prime}}\right]$.

Theorem 4.7. Let $\left(G, \alpha, A\left[\tau_{\Gamma}\right]\right)$ be a pro- $C^{*}$-dynamical system. If $\alpha$ is strongly bounded, then there is a locally Hilbert space $\mathcal{H}$ and a covariant pro- ${ }^{*}$-morphism $\left(i_{A}, i_{G}\right)$ from $\left(G, \alpha, A\left[\tau_{\Gamma}\right]\right)$ to $\mathcal{L}(\mathcal{H})$. Moreover, $i_{A}$ and $i_{G}$ are injective.

Proof. Let $\lambda \in \Lambda$. By Proposition $4.4, \mathcal{R}_{\lambda}\left(G, \alpha, A\left[\tau_{\Gamma}\right]\right)$ is non empty. Let $\left(\varphi^{\lambda}, u^{\lambda}, H_{\lambda}\right)$ be the direct sum of one representative $\left(\varphi, u, H_{\varphi, u}\right)$ of each unitary equivalence class of nondegenerate covariant representations of $\left(G, \alpha, A\left[\tau_{\Gamma}\right]\right)$ from 
$\mathcal{R}_{\lambda}\left(G, \alpha, A\left[\tau_{\Gamma}\right]\right)$. Then $\left(\varphi^{\lambda}, u^{\lambda}, H_{\lambda}\right)$ is a nondegenerate covariant representation of $\left(G, \alpha, A\left[\tau_{\Gamma}\right]\right)$ such that $\left\|\varphi^{\lambda}(a)\right\| \leq p_{\lambda}(a)$ for all $a \in A$.

Let $\mathcal{H}_{\lambda}=\oplus_{\mu \leq \lambda} H_{\mu}$. Then $\mathcal{H}=\lim _{\lambda \rightarrow} \mathcal{H}_{\lambda}$ is a locally Hilbert space. For $a \in A$, the map $i_{A}^{\lambda}(a): \mathcal{H}_{\lambda} \rightarrow \mathcal{H}_{\lambda}$ defined by

$$
i_{A}^{\lambda}(a)\left(\oplus_{\mu \leq \lambda} \xi_{\mu}\right)=\oplus_{\mu \leq \lambda} \varphi^{\mu}(a) \xi_{\mu}
$$

is an element in $L\left(\mathcal{H}_{\lambda}\right)$ and $\left\|i_{A}^{\lambda}(a)\right\| \leq p_{\lambda}(a)$. Moreover, $i_{A}^{\lambda}\left(a^{*}\right)=i_{A}^{\lambda}(a)^{*}$ and $i_{A}^{\lambda}(a b)=i_{A}^{\lambda}(a) i_{A}^{\lambda}(b)$ for all $a, b \in A$. Clearly, $\left(i_{A}^{\lambda}(a)\right)_{\lambda}$ is a direct system of bounded linear operators and $i_{A}(a)=\lim _{\lambda \rightarrow} i_{A}^{\lambda}(a)$ is an element $\mathcal{L}(\mathcal{H})$ such that $i_{A}\left(a^{*}\right)=i_{A}(a)^{*}$ and $i_{A}(a b)=i_{A}(a) i_{A}(b)$ for all $a, b \in A$. Moreover,

$$
p_{\lambda, \mathcal{L}(\mathcal{H})}\left(i_{A}(a)\right)=\left\|i_{A}^{\lambda}(a)\right\| \leq p_{\lambda}(a)
$$

for all $a \in A$ and for all $\lambda \in \Lambda$. Therefore, $i_{A}$ is a pro- $C^{*}$-morphism.

For $t \in G$, the map $i_{G}^{\lambda}(t): \mathcal{H}_{\lambda} \rightarrow \mathcal{H}_{\lambda}$ defined by

$$
i_{G}^{\lambda}(t)\left(\oplus_{\mu \leq \lambda} \xi_{\mu}\right)=\oplus_{\mu \leq \lambda} u^{\mu}(t) \xi_{\mu}
$$

is a unitary element in $L\left(\mathcal{H}_{\lambda}\right)$. Moreover, the map $t \mapsto i_{G}^{\lambda}(t)$ is a unitary *representation of $G$ on $\mathcal{H}_{\lambda}$. Clearly, $\left(i_{G}^{\lambda}(t)\right)_{\lambda}$ is a direct system of unitary operators, and then $i_{G}(t)=\lim _{\lambda \rightarrow} i_{G}^{\lambda}(t)$ is a unitary element $\mathcal{L}(\mathcal{H})$. Moreover, $t \mapsto i_{G}(t)$ is a group morphism from $G$ to the group of unitary operators on $\mathcal{H}$, and since for each $\xi \in \mathcal{H}$, the map $t \mapsto i_{G}(t) \xi$ from $G$ to $\mathcal{H}$ is continuous, $t \mapsto i_{G}(t)$ is a unitary *-representation of $G$ on $\mathcal{H}$. We have

$$
\begin{aligned}
i_{A}\left(\alpha_{t}(a)\right)\left(\oplus_{\mu \leq \lambda} \xi_{\mu}\right) & =i_{A}^{\lambda}\left(\alpha_{t}(a)\right)\left(\oplus_{\mu \leq \lambda} \xi_{\mu}\right)=\oplus_{\mu \leq \lambda} \varphi^{\mu}\left(\alpha_{t}(a)\right)\left(\xi_{\mu}\right) \\
& =\oplus_{\mu \leq \lambda} u^{\mu}(t) \varphi^{\mu}(a) u^{\mu}(t)^{*}\left(\xi_{\mu}\right) \\
& =i_{G}(t) i_{A}(a) i_{G}(t)^{*}\left(\oplus_{\mu \leq \lambda} \xi_{\mu}\right)
\end{aligned}
$$

for all $a \in A$, for all $t \in G$ and for all $\oplus_{\mu \leq \lambda} \xi_{\mu} \in \mathcal{H}_{\lambda}, \lambda \in \Lambda$, and so

$$
i_{A}\left(\alpha_{t}(a)\right)=i_{G}(t) i_{A}(a) i_{G}(t)^{*}
$$

for all $a \in A$ and for all $t \in G$.

Suppose that $i_{A}(a)=0$. Then $i_{A}^{\lambda}(a)=0$ for all $\lambda \in \Lambda$ and so $\varphi(a)=0$ for all nondegenerate covariant representation $\left(\varphi, u, H_{\varphi, u}\right)$ of $\left(G, \alpha, A\left[\tau_{\Gamma}\right]\right)$. By Proposition 4.4 and Remark 4.5, $\psi(a)=0$ for all representations $\psi$ of $A$. Therefore, $p_{\lambda}(a)=0$ for all $\lambda \in \Lambda$, and then $a=0$.

Suppose that $i_{G}(t)=\mathrm{id}_{\mathcal{H}}$. Then $i_{G}^{\lambda}(t)=\operatorname{id}_{\mathcal{H}_{\lambda}}$ for all $\lambda \in \Lambda$, and so $u(t)=\operatorname{id}_{H_{\varphi, u}}$ for all nondegenerate covariant representation $\left(\varphi, u, H_{\varphi, u}\right)$ of $\left(G, \alpha, A\left[\tau_{\Gamma}\right]\right)$, whence we deduce that $t=e$.

The following proposition gives a characterization of inverse limit actions.

Proposition 4.8. Let $\left(G, \alpha, A\left[\tau_{\Gamma}\right]\right)$ be a pro- $C^{*}$-dynamical system. Then the following statements are equivalent.

(1) $\alpha$ is an inverse limit action.

(2) There is a locally Hilbert space $\mathcal{H}$ and a covariant pro- ${ }^{*}{ }^{*}$ morphism $\left(i_{A}, i_{G}\right)$ from $\left(G, \alpha, A\left[\tau_{\Gamma}\right]\right)$ to $\mathcal{L}(\mathcal{H})$ such that $p_{\lambda, \mathcal{L}(\mathcal{H})}\left(i_{A}(a)\right)=p_{\lambda}(a)$ for all $\lambda \in \Lambda$ and $a \in A$. 
Proof. $(1) \Rightarrow(2)$ See [J3, Proposition 3.1] and [I, Theorem 5.1].

$(2) \Rightarrow(1)$ From

$$
i_{A}\left(\alpha_{t}(a)\right)=i_{G}(t) i_{A}(a) i_{G}(t)^{*}
$$

for all $t \in G$ and for all $a \in A$, and taking into account that $i_{G}(t)$ is a unitary element in $\mathcal{L}(\mathcal{H})$ for all $t \in G$, we deduce that

$$
\begin{aligned}
p_{\lambda}\left(\alpha_{t}(a)\right) & =p_{\lambda, \mathcal{L}(\mathcal{H})}\left(i_{A}\left(\alpha_{t}(a)\right)\right)=p_{\lambda, \mathcal{L}(\mathcal{H})}\left(i_{G}(t) i_{A}(a) i_{G}(t)^{*}\right) \\
& =p_{\lambda, \mathcal{L}(\mathcal{H})}\left(i_{A}(a)\right)=p_{\lambda}(a)
\end{aligned}
$$

for all $t \in G$, for all $a \in A$ and for all $t \in G$. Therefore, $\alpha$ is an inverse limit action.

\section{The FUlL PRO- $C^{*}$-CROSSED PRODUCT}

Let $\left(G, \alpha, A\left[\tau_{\Gamma}\right]\right)$ be a pro- $C^{*}$-dynamical system and let $B\left[\tau_{\Gamma^{\prime}}\right]$ be a pro- $C^{*}$ algebra whose topology is given by the family of $C^{*}$-seminorms $\Gamma^{\prime}=\left\{q_{\delta}\right\}_{\delta \in \Delta}$.

If $u$ is a strict continuous group morphism from $G$ to $\mathcal{U}\left(M\left(B\left[\tau_{\Gamma^{\prime}}\right]\right)\right)$, then there is a *-morphism $u: C_{c}(G) \rightarrow M\left(B\left[\tau_{\Gamma^{\prime}}\right]\right)$ given by $u(f)=\int_{G} f(s) u_{s} d s$, where $d s$ denotes the Haar measure on $G$ (see [J2]).

Definition 5.1. Let $\left(G, \alpha, A\left[\tau_{\Gamma}\right]\right)$ be a pro- $C^{*}$-dynamical system. A pro- $C^{*}$ algebra, denoted by $G \times{ }_{\alpha} A\left[\tau_{\Gamma}\right]$, together with a covariant pro- $C^{*}$-morphism $\left(\iota_{A}, \iota_{G}\right)$ from $\left(G, \alpha, A\left[\tau_{\Gamma}\right]\right)$ to $G \times_{\alpha} A\left[\tau_{\Gamma}\right]$ which verifies the following:

(1) for each nondegenerate covariant representation $(\varphi, u, \mathcal{H})$ of $\left(G, \alpha, A\left[\tau_{\Gamma}\right]\right)$, there is a nondegenerate representation $(\Phi, \mathcal{H})$ of $G \times{ }_{\alpha} A\left[\tau_{\Gamma}\right]$ such that $\bar{\Phi} \circ \iota_{A}=\varphi$ and $\bar{\Phi} \circ \iota_{G}=u$;

(2) $\overline{\operatorname{span}\left\{\iota_{A}(a) \iota_{G}(f) ; a \in A, f \in C_{c}(G)\right\}}=G \times_{\alpha} A\left[\tau_{\Gamma}\right]$;

is called the full pro- $C^{*}$-crossed product of $A\left[\tau_{\Gamma}\right]$ by $\alpha$.

Remark 5.2. The covariant morphism $\left(\iota_{A}, \iota_{G}\right)$ from the above definition is nondegenerate.

Proposition 5.3. Let $\left(G, \alpha, A\left[\tau_{\Gamma}\right]\right)$ be a pro- $C^{*}$-dynamical system such that there is a full pro- $C^{*}$-crossed product of $A\left[\tau_{\Gamma}\right]$ by $\alpha$ and $(\varphi, u)$ a nondegenerate covariant morphism from $\left(G, \alpha, A\left[\tau_{\Gamma}\right]\right)$ to a pro- $C^{*}$-algebra $B\left[\tau_{\Gamma^{\prime}}\right]$. Then there is a unique nondegenerate pro- $C^{*}$-morphism $\varphi \times u: G \times{ }_{\alpha} A\left[\tau_{\Gamma}\right] \rightarrow M\left(B\left[\tau_{\Gamma^{\prime}}\right]\right)$ such that

$$
\overline{\varphi \times u} \circ \iota_{A}=\varphi \text { and } \overline{\varphi \times u} \circ \iota_{G}=u \text {. }
$$

Moreover, the $\operatorname{map}(\varphi, u) \rightarrow \varphi \times u$ is a bijection between nondegenerate covariant morphisms of $\left(G, \alpha, A\left[\tau_{\Gamma}\right]\right)$ onto nondegenerate morphisms of $G \times{ }_{\alpha} A\left[\tau_{\Gamma}\right]$.

Proof. Let $q_{\delta} \in \Gamma^{\prime}$ and $\left(\psi_{\delta}, \mathcal{H}\right)$ a faithful nondegenerate representation of $B_{\delta}$. Then, $\left(\overline{\psi_{\delta}} \circ \overline{\pi_{\delta}^{B}} \circ \varphi, \overline{\psi_{\delta}} \circ \overline{\pi_{\delta}^{B}} \circ u, \mathcal{H}\right)$ is a nondegenerate covariant representation of $\left(G, \alpha, A\left[\tau_{\Gamma}\right]\right)$, and by Definition 5.1 , there is a nondegenerate representation $\left(\phi_{\delta}, \mathcal{H}\right)$ of $G \times{ }_{\alpha} A\left[\tau_{\Gamma}\right]$ such that

$$
\overline{\phi_{\delta}} \circ \iota_{A}=\overline{\psi_{\delta}} \circ \overline{\pi_{\delta}^{B}} \circ \varphi \text { and } \overline{\phi_{\delta}} \circ \iota_{G}=\overline{\psi_{\delta}} \circ \overline{\pi_{\delta}^{B}} \circ u \text {. }
$$


Let $\Phi_{\delta}=\overline{\psi_{\delta}^{-1}} \circ \phi_{\delta}$. Then $\Phi_{\delta}$ is a nondegenerate pro- $C^{*}$-morphism from $G \times{ }_{\alpha} A\left[\tau_{\Gamma}\right]$ to $M\left(B_{\delta}\right)$. Moreover, for $q_{\delta_{1}}, q_{\delta_{2}} \in \Gamma^{\prime}$ with $q_{\delta_{1}} \geq q_{\delta_{2}}$, we have $\overline{\pi_{\delta_{1} \delta_{2}}^{B}} \circ \Phi_{\delta_{1}}=\Phi_{\delta_{2}}$. Therefore, there is a nondegenerate pro- $C^{*}$-morphism $\varphi \times u: G \times{ }_{\alpha} A\left[\tau_{\Gamma}\right] \rightarrow$ $M\left(B\left[\tau_{\Gamma^{\prime}}\right]\right)$ such that

$$
\overline{\pi_{\delta}^{B}} \circ \varphi \times u=\Phi_{\delta}
$$

for all $q_{\delta} \in \Gamma^{\prime}$. Moreover, $\overline{\varphi \times u} \circ \iota_{A}=\varphi$ and $\overline{\varphi \times u} \circ \iota_{G}=u$, and since $\left\{\iota_{A}(a) \iota_{G}(f) ; a \in A, f \in C_{c}(G)\right\}$ generates $G \times{ }_{\alpha} A\left[\tau_{\Gamma}\right], \varphi \times u$ is unique with the above properties.

Let $\Phi: G \times{ }_{\alpha} A\left[\tau_{\Gamma}\right] \rightarrow M\left(B\left[\tau_{\Gamma^{\prime}}\right]\right)$ be a nondegenerate pro- $C^{*}$-morphism. Then $\varphi=\bar{\Phi} \circ \iota_{A}$ is a nondegenerate pro- $C^{*}$-morphism from $A\left[\tau_{\Gamma}\right]$ to $M\left(B\left[\tau_{\Gamma^{\prime}}\right]\right)$ and $u=$ $\bar{\Phi} \circ \iota_{G}$ is a strict continuous morphism from $G$ to $\mathcal{U}\left(M\left(B\left[\tau_{\Gamma^{\prime}}\right]\right)\right)$, since $\iota_{G}$ is a strict continuous morphism from $G$ to $M\left(G \times{ }_{\alpha} A\left[\tau_{\Gamma}\right]\right)$ and $\bar{\Phi}$ is strongly continuous on the bounded subsets of $M\left(G \times_{\alpha} A\left[\tau_{\Gamma}\right]\right)$. Moreover, $(\varphi, u)$ is a nondegenerate covariant morphism from $A\left[\tau_{\Gamma}\right]$ to $B\left[\tau_{\Gamma^{\prime}}\right]$, and $\varphi \times u=\Phi$. If $(\psi, v)$ is another nondegenerate covariant morphism from $A\left[\tau_{\Gamma}\right]$ to $B\left[\tau_{\Gamma^{\prime}}\right]$ such that $\psi \times v=\Phi$, then $\psi=\bar{\Phi} \circ \iota_{A}=\varphi$ and $v=\bar{\Phi} \circ \iota_{G}=u$.

The following corollary provides uniqueness of the full pro- $C^{*}$-crossed product.

Corollary 5.4. Let $\left(G, \alpha, A\left[\tau_{\Gamma}\right]\right)$ be a pro- $C^{*}$-dynamical system such that there is a full pro- $C^{*}$-crossed product of $A\left[\tau_{\Gamma}\right]$ by $\alpha$. Then the full pro- $C^{*}$-crossed product of $A\left[\tau_{\Gamma}\right]$ by $\alpha$ is unique up to a pro- $C^{*}$-isomorphism.

Proof. Let $B\left[\tau_{\Gamma^{\prime}}\right]$ be a pro- $C^{*}$-algebra and $\left(j_{A}, j_{G}\right)$ a covariant pro- $C^{*}$-morphism from $\left(G, \alpha, A\left[\tau_{\Gamma}\right]\right)$ to $B\left[\tau_{\Gamma^{\prime}}\right]$ which satisfy the relations $(1)-(2)$ from Definition 5.1. Then, by Proposition 5.3, there is a nondegenerate pro- $C^{*}$-morphism $\Phi: G \times_{\alpha} A\left[\tau_{\Gamma}\right] \rightarrow M\left(B\left[\tau_{\Gamma^{\prime}}\right]\right)$ such that $\bar{\Phi} \circ \iota_{A}=j_{A}$ and $\bar{\Phi} \circ \iota_{G}=j_{G}$. Since $\left\{\iota_{A}(a) \iota_{G}(f) ; a \in A, f \in C_{c}(G)\right\}$ generates $G \times{ }_{\alpha} A\left[\tau_{\Gamma}\right]$ and $\left\{j_{A}(a) j_{G}(f) ; a \in A\right.$, $\left.f \in C_{c}(G)\right\}$ generates $B\left[\tau_{\Gamma^{\prime}}\right], \Phi\left(G \times{ }_{\alpha} A\left[\tau_{\Gamma}\right]\right) \subseteq B$.

In the same way, there is a pro- $C^{*}$-morphism $\Psi: B\left[\tau_{\Gamma^{\prime}}\right] \rightarrow G \times{ }_{\alpha} A\left[\tau_{\Gamma}\right]$ such that $\bar{\Psi} \circ j_{A}=\iota_{A}$ and $\bar{\Psi} \circ j_{G}=\iota_{G}$. From these facts and Definition 5.1 (2), we deduce that $\Phi \circ \Psi=\mathrm{id}_{B}$ and $\Psi \circ \Phi=\mathrm{id}_{G \times_{\alpha} A\left[\tau_{\Gamma}\right]}$, and so $\Phi$ is a pro- $C^{*}$-isomorphism.

The following proposition relates the nondegenerate covariant representations of a pro- $C^{*}$-dynamical system $\left(G, \alpha, A\left[\tau_{\Gamma}\right]\right)$ with the nondegenerate representations of the full pro- $C^{*}$-crossed product of $A\left[\tau_{\Gamma}\right]$ by $\alpha$.

Proposition 5.5. Let $\left(G, \alpha, A\left[\tau_{\Gamma}\right]\right)$ be a pro- $C^{*}$-dynamical system such that there is the full pro- $C^{*}$-crossed product of $A\left[\tau_{\Gamma}\right]$ by $\alpha$. Then there is a bijective correspondence between nondegenerate covariant representations of $\left(G, \alpha, A\left[\tau_{\Gamma}\right]\right)$ and nondegenerate representations of $G \times{ }_{\alpha} A\left[\tau_{\Gamma}\right]$.

Proof. Let $(\varphi, u, \mathcal{H})$ be a nondegenerate covariant representation of $\left(G, \alpha, A\left[\tau_{\Gamma}\right]\right)$. Then, by Definition 5.1, there is a nondegenerate representation $(\varphi \times u, \mathcal{H})$ of $G \times{ }_{\alpha} A\left[\tau_{\Gamma}\right]$ such that $\overline{\varphi \times u} \circ \iota_{A}=\varphi$ and $\overline{\varphi \times u} \circ \iota_{G}=u$. Moreover, by Definition $5.1(2),(\varphi \times u, \mathcal{H})$ is unique, and since $\varphi$ is nondegenerate, it is nondegenerate too. 
Let $(\Phi, \mathcal{H})$ be a nondegenerate representation of $G \times{ }_{\alpha} A\left[\tau_{\Gamma}\right]$. Then $\left(\bar{\Phi} \circ \iota_{A}\right.$, $\left.\bar{\Phi} \circ \iota_{G}, \mathcal{H}\right)$ is a covariant representation of $\left(G, \alpha, A\left[\tau_{\Gamma}\right]\right)$, and moreover, $\left(\bar{\Phi} \circ \iota_{A}\right) \times$ $\left(\bar{\Phi} \circ \iota_{G}\right)=\Phi$. Since $\iota_{A}$ and $\Phi$ are nondegenerate, the net $\left\{\bar{\Phi}\left(\iota_{A}\left(e_{i}\right)\right)\right\}_{i}$, where $\left\{e_{i}\right\}_{i}$ is an approximate unit of $A\left[\tau_{\Gamma}\right]$, converges strictly to $\operatorname{id}_{\mathcal{H}}$, and so $\bar{\Phi} \circ \iota_{A}$ is nondegenerate.

Suppose that there is another nondegenerate covariant representation $(\varphi, u, \mathcal{H})$ of $\left(G, \alpha, A\left[\tau_{\Gamma}\right]\right)$ such that $\varphi \times u=\Phi$. Then $\varphi=\overline{\varphi \times u} \circ \iota_{A}=\bar{\Phi} \circ \iota_{A}$ and $u=\overline{\varphi \times} u \circ \iota_{G}=\bar{\Phi} \circ \iota_{G}$. Therefore, the map $(\varphi, u, \mathcal{H}) \mapsto(\varphi \times u, \mathcal{H})$ is bijective.

Theorem 5.6. Let $\left(G, \alpha, A\left[\tau_{\Gamma}\right]\right)$ be a pro- $C^{*}$-dynamical system such that $\alpha$ is strongly bounded. Then, there is the full pro- $C^{*}$-crossed product of $A\left[\tau_{\Gamma}\right]$ by $\alpha$.

Proof. By Theorem 4.7, there is a locally Hilbert space $\mathcal{H}$ and a covariant pro$C^{*}$-morphism $\left(i_{A}, i_{G}\right)$ from $A\left[\tau_{\Gamma}\right]$ to $\mathcal{L}(\mathcal{H})$.

Let $B=\overline{\operatorname{span}\left\{i_{A}(a) i_{G}(f) ; a \in A, f \in C_{c}(G)\right\}} \subseteq \mathcal{L}(\mathcal{H})$. To show that $B$ is a pro- $C^{*}$-algebra, we must show that $B$ is closed under taking adjoints and multiplication. For this, since $B=\lim _{\longleftarrow_{\lambda}} \overline{\pi_{\lambda}^{\mathcal{H}}(B)}$ ([M, Chapter III, Theorem 3.1]), it is sufficient to show that for each $\lambda \in \Lambda, \pi_{\lambda}^{\mathcal{H}}\left(i_{A}(b) i_{G}(f) i_{A}(a) i_{G}(h)\right)$ and $\pi_{\lambda}^{\mathcal{H}}\left(i_{G}(f) i_{A}(a)\right)$ are elements in the closure of $\pi_{\lambda}^{\mathcal{H}}(B)$ in $L\left(\mathcal{H}_{\lambda}\right)$ for all $a, b \in A$ and for all $f, h \in C_{c}(G)$.

The map $s \rightarrow \pi_{\lambda}^{A}\left(f(s) \alpha_{s}(a)\right)$ from $G$ to $A_{\lambda}$ defines an element in $C_{c}\left(G, A_{\lambda}\right)$, and so there is a net $\left\{\pi_{\lambda}^{A}\left(a_{j}\right) \otimes f_{j}\right\}_{j \in J}$ in $A_{\lambda} \otimes_{\text {alg }} C_{c}(G)$ with $\operatorname{supp} f_{j}, \operatorname{supp} f \subseteq K$ for some compact subset $K$, which converges uniformly to this map.

By [J4, Lemma 3.7],

$$
\begin{aligned}
\pi_{\lambda}^{\mathcal{H}}\left(i_{G}(f) i_{A}(a)\right) & =\int_{G} f(s) i^{\lambda}(s) d s \pi_{\lambda}^{\mathcal{H}}\left(i_{A}(a)\right)=\int_{G} f(s) \pi_{\lambda}^{\mathcal{H}}\left(i_{G}(s) i_{A}(a)\right) d s \\
& =\int_{G} f(s) \pi_{\lambda}^{\mathcal{H}}\left(i_{A}\left(\alpha_{s}(a)\right) i_{G}(s)\right) d s \\
& =\int_{G} f(s) i_{A}^{\lambda}\left(\alpha_{s}(a)\right) i_{G}^{\lambda}(s) d s
\end{aligned}
$$

and

$$
\pi_{\lambda}^{\mathcal{H}}\left(i_{A}\left(a_{j}\right) i_{G}\left(f_{j}\right)\right)=\pi_{\lambda}^{\mathcal{H}}\left(i_{A}\left(a_{j}\right)\right) \int_{G} f_{j}(s) i_{G}^{\lambda}(s) d s=\int_{G} i_{A}^{\lambda}\left(a_{j}\right) f_{j}(s) i_{G}^{\lambda}(s) d s
$$


for each $j \in J$. Then, we have

$$
\begin{aligned}
& \left\|\pi_{\lambda}^{\mathcal{H}}\left(i_{G}(f) i_{A}(a)\right)-\pi_{\lambda}^{\mathcal{H}}\left(i_{A}\left(a_{j}\right) i_{G}\left(f_{j}\right)\right)\right\|_{L\left(\mathcal{H}_{\lambda}\right)} \\
\leq & \int_{G}\left\|f(s) i_{A}^{\lambda}\left(\alpha_{s}(a)\right) i_{G}^{\lambda}(s)-i_{A}^{\lambda}\left(a_{j}\right) f_{j}(s) i_{G}^{\lambda}(s)\right\|_{L\left(\mathcal{H}_{\lambda}\right)} d s \\
\leq & M \sup \left\{\left\|f(s) i_{A}^{\lambda}\left(\alpha_{s}(a)\right) i_{G}^{\lambda}(s)-i_{A}^{\lambda}\left(a_{j}\right) f_{j}(s) i_{G}^{\lambda}(s)\right\|_{L\left(\mathcal{H}_{\lambda}\right)}, s \in K\right\} \\
= & M \sup \left\{\left\|i_{A}^{\lambda}\left(f(s) \alpha_{s}(a)-f_{j}(s) a_{j}\right)\right\|_{L\left(\mathcal{H}_{\lambda}\right)}\left\|i_{G}^{\lambda}(s)\right\|_{L\left(\mathcal{H}_{\lambda}\right)}, s \in K\right\} \\
\leq & M \sup \left\{p_{\lambda}\left(f(s) \alpha_{s}(a)-f_{j}(s) a_{j}\right), s \in K\right\} \\
= & M \sup \left\{\left\|\pi_{\lambda}^{A}\left(f(s) \alpha_{s}(a)\right)-f_{j}(s) \pi_{\lambda}^{A}\left(a_{j}\right)\right\|_{A_{\lambda}}, s \in K\right\}
\end{aligned}
$$

for all $j \in J$, where $M=\int_{K} d g$, and so $\pi_{\lambda}^{\mathcal{H}}\left(i_{G}(f) i_{A}(a)\right) \in \overline{\pi_{\lambda}^{\mathcal{H}}(B)}$.

On the other hand,

$$
\begin{aligned}
& \left\|\pi_{\lambda}^{\mathcal{H}}\left(i_{A}(b) i_{G}(f) i_{A}(a) i_{G}(h)\right)-\pi_{\lambda}^{\mathcal{H}}\left(i_{A}\left(b a_{j}\right) i_{G}\left(f_{j} * h\right)\right)\right\|_{L\left(\mathcal{H}_{\lambda}\right)} \\
= & \left\|\pi_{\lambda}^{\mathcal{H}}\left(i_{A}(b) i_{G}(f) i_{A}(a) i_{G}(h)-i_{A}(b) i_{A}\left(a_{j}\right) i_{G}\left(f_{j}\right) i_{G}(h)\right)\right\|_{L\left(\mathcal{H}_{\lambda}\right)} \\
\leq & \left\|\pi_{\lambda}^{\mathcal{H}}\left(i_{A}(b)\right) \pi_{\lambda}^{\mathcal{H}}\left(i_{G}(f) i_{A}(a)-i_{A}\left(a_{j}\right) i_{G}\left(f_{j}\right)\right) \pi_{\lambda}^{\mathcal{H}}\left(i_{G}(h)\right)\right\|_{L\left(\mathcal{H}_{\lambda}\right)} \\
\leq & \left\|\pi_{\lambda}^{\mathcal{H}}\left(i_{A}(b)\right)\right\|_{L\left(\mathcal{H}_{\lambda}\right)}\left\|\pi_{\lambda}^{\mathcal{H}}\left(i_{G}(h)\right)\right\|_{L\left(\mathcal{H}_{\lambda}\right)} \\
& \left\|\pi_{\lambda}^{\mathcal{H}}\left(i_{G}(f) i_{A}(a)\right)-\pi_{\lambda}^{\mathcal{H}}\left(i_{A}\left(a_{j}\right) i_{G}\left(f_{j}\right)\right)\right\|_{L\left(\mathcal{H}_{\lambda}\right)}
\end{aligned}
$$

whence, we deduce that $\pi_{\lambda}^{\mathcal{H}}\left(i_{A}(b) i_{G}(f) i_{A}(a) i_{G}(h)\right) \in \overline{\pi_{\lambda}^{\mathcal{H}}(B)}$. Thus, we showed that $\pi_{\lambda}^{\mathcal{H}}\left(i_{G}(f) i_{A}(a)\right), \pi_{\lambda}^{\mathcal{H}}\left(i_{A}(b) i_{G}(f) i_{A}(a) i_{G}(h)\right) \in \overline{\pi_{\lambda}^{\mathcal{H}}(B)}$ for each $\lambda \in \Lambda$, and so $i_{G}(f) i_{A}(a), i_{A}(b) i_{G}(f) i_{A}(a) i_{G}(h) \in B$. Therefore, $B$ is a pro- $C^{*}$-algebra.

In the same manner, we show that for each $a \in A, i_{A}(a) i_{A}(b) i_{G}(f) \in B$ and $i_{A}(b) i_{G}(f) i_{A}(a) \in B$ for all $b \in A$ and for all $f \in C_{c}(G)$, and so $i_{A}(a) \in M(B)$.

From,

$$
i_{G}(t) i_{A}(a) i_{G}(f)=\int_{G} f(s) i_{A}\left(\alpha_{t}(a)\right) i_{G}(t s) d s \in B
$$

and

$$
i_{G}(f) i_{A}(a) i_{G}(t)=\int_{G} f(s) i_{A}\left(\alpha_{s}(a)\right) i_{G}(s t) d s \in B
$$

for all $a \in A$, for all $f \in C_{c}(G)$ and for all $t \in G$, we deduce that $i_{G}(t) \in M(B)$ for all $t \in G$.

Let $\left(\psi, v, H_{\psi, v}\right)$ be a nondegenerate covariant representation of $\left(G, \alpha, A\left[\tau_{\Gamma}\right]\right)$. Then there is $\left(\varphi, u, H_{\varphi, u}\right) \in \mathcal{R}_{\lambda}\left(G, \alpha, A\left[\tau_{\Gamma}\right]\right)$ such that $\left(\psi, v, H_{\psi, v}\right)$ and $\left(\varphi, u, H_{\varphi, u}\right)$ are unitarily equivalent. So there is a unitary operator $U: H_{\psi, v} \rightarrow H_{\varphi, u}$ such that $\psi(a)=U^{*} \varphi(a) U$ for all $a \in A$ and $v_{t}=U^{*} u_{t} U$ for all $t \in G$. The map $\Psi: \mathcal{L}(\mathcal{H}) \rightarrow L\left(H_{\lambda}\right)$ given by

$$
\Psi(T)=\left.\pi_{\lambda}^{\mathcal{H}}(T)\right|_{H_{\lambda}}
$$

is a representation of $\mathcal{L}(\mathcal{H})$ on $H_{\lambda}$ (see the proof of Theorem 4.7). From

$$
\Psi\left(i_{A}(a)\right)\left(H_{\varphi, u}\right)=i_{A}^{\lambda}(a)\left(H_{\varphi, u}\right) \subseteq H_{\varphi, u}
$$


for all $a \in A$ and

$$
\Psi\left(i_{G}(t)\right)\left(H_{\varphi, u}\right)=i_{G}^{\lambda}(t)\left(H_{\varphi, u}\right) \subseteq H_{\varphi, u}
$$

for all $t \in G$, and taking into account that $B$ is generated by $\left\{i_{A}(a) i_{G}(f) ; a \in\right.$ $\left.A, f \in C_{c}(G)\right\}$, we deduce that $\Psi(B)\left(H_{\varphi, u}\right) \subseteq H_{\varphi, u}$. Let $\Phi: B \rightarrow L\left(H_{\psi, v}\right)$ given by

$$
\Phi(b)=\left.U^{*} \Psi(b)\right|_{H_{\varphi, u}} U .
$$

Clearly, $\Phi$ is a nondegenerate representation of $B$ on $H_{\psi, v}$,

$$
\bar{\Phi}\left(i_{A}(a)\right)=\left.U^{*} \Psi\left(i_{A}(a)\right)\right|_{H_{\varphi, u}} U=\left.U^{*} i_{A}^{\lambda}(a)\right|_{H_{\varphi, u}} U=U^{*} \varphi(a) U=\psi(a)
$$

for all $a \in A$, and

$$
\bar{\Phi}\left(i_{G}(t)\right)=\left.U^{*} \Psi\left(i_{G}(t)\right)\right|_{H_{\varphi, u}} U=\left.U^{*} i_{G}^{\lambda}(t)\right|_{H_{\varphi, u}} U=U^{*} u_{t} U=v_{t}
$$

for all $t \in G$.

Remark 5.7. The index of the family of seminorms which gives the topology on the full pro- $C^{*}$-crossed product of $A\left[\tau_{\Gamma}\right]$ by $\alpha$ is the same with the index of the family of seminorms which gives the topology on $A\left[\tau_{\Gamma}\right]$.

Proposition 5.8. Let $\left(G, \alpha, A\left[\tau_{\Gamma}\right]\right)$ be a pro- $C^{*}$-dynamical system such that $\alpha$ is an inverse limit action. Then for each $\lambda \in \Lambda$, the $C^{*}$-algebra $\left(G \times_{\alpha} A\left[\tau_{\Gamma}\right]\right)_{\lambda}$ is isomorphic to the full $C^{*}$-crossed product of $A_{\lambda}$ by $\alpha^{\lambda}$.

Proof. By Theorem 4.7, Proposition 4.8 and Corollary 5.4, there is a $C^{*}$-morphism $i_{A_{\lambda}}: A_{\lambda} \rightarrow M\left(\left(G \times_{\alpha} A\left[\tau_{\Gamma}\right]\right)_{\lambda}\right)$ such that $i_{A_{\lambda}} \circ \pi_{\lambda}^{A}=\overline{\pi_{\lambda}^{G \times_{\alpha} A\left[\tau_{\Gamma}\right]}} \circ i_{A}$. Using the fact

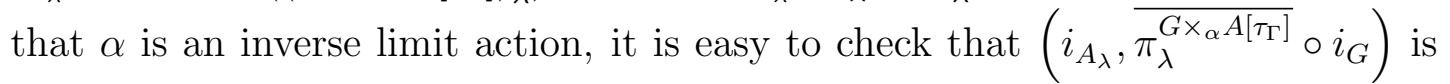
a covariant $C^{*}$-morphism from $\left(G, \alpha^{\lambda}, A_{\lambda}\right)$ to $\left(G \times{ }_{\alpha} A\left[\tau_{\Gamma}\right]\right)_{\lambda}$. Moreover,

$$
\begin{aligned}
& \overline{\operatorname{span}\left\{i_{A_{\lambda}}\left(\pi_{\lambda}^{A}(a)\right) \overline{\pi_{\lambda}^{G \times_{\alpha} A\left[\tau_{\Gamma}\right]}}\left(i_{G}(f)\right) ; a \in A, f \in C_{c}(G)\right\}} \\
& =\overline{\operatorname{span}\left\{\overline{\pi_{\lambda}^{G \times_{\alpha} A\left[\tau_{\Gamma}\right]}}\left(i_{A}(a) i_{G}(f)\right) ; a \in A, f \in C_{c}(G)\right\}} \\
& =\overline{\pi_{\lambda}^{G \times{ }_{\alpha} A\left[\tau_{\Gamma}\right]}}\left(G \times{ }_{\alpha} A\left[\tau_{\Gamma}\right]\right)=\left(G \times_{\alpha} A\left[\tau_{\Gamma}\right]\right)_{\lambda} .
\end{aligned}
$$

Let $(\varphi, u, \mathcal{H})$ be a nondegenerate covariant representation of $\left(G, \alpha^{\lambda}, A_{\lambda}\right)$. Then $\left(\varphi \circ \pi_{\lambda}^{A}, u, \mathcal{H}\right)$ is a nondegenerate covariant representation of $\left(G, \alpha, A\left[\tau_{\Gamma}\right]\right)$ and by Definition 5.1, there is a nondegenerate representation $(\Phi, \mathcal{H})$ of $G \times{ }_{\alpha} A\left[\tau_{\Gamma}\right]$ such that $\bar{\Phi} \circ i_{A}=\varphi \circ \pi_{\lambda}^{A}$ and $\bar{\Phi} \circ i_{G}=u$. Moreover, by the proof of Theorem 5.6,

$$
\|\Phi(b)\| \leq p_{\lambda, G \times{ }_{\alpha} A\left[\tau_{\Gamma}\right]}(b)
$$

for all $b \in G \times{ }_{\alpha} A\left[\tau_{\Gamma}\right]$. Therefore, there is the $C^{*}$-morphism $\Phi_{\lambda}:\left(G \times{ }_{\alpha} A\left[\tau_{\Gamma}\right]\right)_{\lambda} \rightarrow$ $L(\mathcal{H})$ such that $\Phi_{\lambda} \circ \overline{\pi_{\lambda}^{G \times_{\alpha} A\left[\tau_{\Gamma}\right]}}=\Phi$. Moreover, $\left(\Phi_{\lambda}, \mathcal{H}\right)$ is a nondegenerate representation of $\left(G \times{ }_{\alpha} A\left[\tau_{\Gamma}\right]\right)_{\lambda}$ such that

$$
\bar{\Phi}_{\lambda} \circ i_{A_{\lambda}}=\varphi \text { and } \overline{\Phi_{\lambda}} \circ\left(\overline{\pi_{\lambda}^{G \times_{\alpha} A\left[\tau_{\Gamma}\right]}} \circ i_{G}\right)=u \text {. }
$$

Thus, we showed that $\left(G \times{ }_{\alpha} A\left[\tau_{\Gamma}\right]\right)_{\lambda}$ is isomorphic to $G \times{ }_{\alpha^{\lambda}} A_{\lambda}$. 
Corollary 5.9. Let $\left(G, \alpha, A\left[\tau_{\Gamma}\right]\right)$ be a pro- $C^{*}$-dynamical system such that $\alpha$ is an inverse limit action. Then the pro- $C^{*}$-algebras $G \times_{\alpha} A\left[\tau_{\Gamma}\right]$ and $\lim _{\leftarrow \lambda} G \times_{\alpha^{\lambda}} A_{\lambda}$ are isomorphic.

Remark 5.10. If $\left(G, \alpha, A\left[\tau_{\Gamma}\right]\right)$ is a pro- $C^{*}$-dynamical system such that $\alpha$ is an inverse limit action, then the notion of full pro- $C^{*}$-crossed product in the sense of Definition 5.1 coincides to the notion of full crossed product introduced by $[\mathrm{P} 2, \mathrm{~J} 4]$.

Definition 5.11. We say that $\left(G, \alpha, A\left[\tau_{\Gamma}\right]\right)$ and $\left(G, \beta, B\left[\tau_{\Gamma^{\prime}}\right]\right)$ are conjugate if there is a pro- $C^{*}$-isomorphism $\varphi: A\left[\tau_{\Gamma}\right] \rightarrow B\left[\tau_{\Gamma^{\prime}}\right]$ such that $\varphi \circ \alpha_{t}=\beta_{t} \circ \varphi$ for all $t \in G$.

Remark 5.12. If $\left(G, \alpha, A\left[\tau_{\Gamma}\right]\right)$ and $\left(G, \beta, B\left[\tau_{\Gamma^{\prime}}\right]\right)$ are conjugate and $\alpha$ is strongly bounded, then $\beta$ is strongly bounded too.

Proposition 5.13. Let $\left(G, \alpha, A\left[\tau_{\Gamma}\right]\right)$ and $\left(G, \beta, B\left[\tau_{\Gamma^{\prime}}\right]\right)$ be two pro- $C^{*}$-dynamical systems such that $\alpha$ and $\beta$ are strongly bounded. If $\left(G, \alpha, A\left[\tau_{\Gamma}\right]\right)$ and $\left(G, \beta, B\left[\tau_{\Gamma^{\prime}}\right]\right)$ are conjugate, then the full pro- $C^{*}$-crossed products associated to these pro- $C^{*}$ dynamical systems are isomorphic.

Proof. Let $\varphi: A\left[\tau_{\Gamma}\right] \rightarrow B\left[\tau_{\Gamma^{\prime}}\right]$ be a pro- $C^{*}$-isomorphism such that $\varphi \circ \alpha_{t}=\beta_{t} \circ \varphi$ for all $t \in G$. It is easy to check that $\left(\iota_{B} \circ \varphi, \iota_{G, B}\right)$ is a nondegenerate covariant morphism from $\left(G, \alpha, A\left[\tau_{\Gamma}\right]\right)$ to $G \times_{\beta} B\left[\tau_{\Gamma^{\prime}}\right]$, where $\left(\iota_{B}, \iota_{G, B}\right)$ is the covariant morphism from $\left(G, \beta, B\left[\tau_{\Gamma^{\prime}}\right]\right)$ to $G \times{ }_{\beta} B\left[\tau_{\Gamma^{\prime}}\right]$ which defines the full pro- $C^{*}$-crossed product of $B\left[\tau_{\Gamma^{\prime}}\right]$ by $\beta$. Then, by Proposition 5.3, there is a nondegenerate pro$C^{*}$-morphism $\Phi: G \times_{\alpha} A\left[\tau_{\Gamma}\right] \rightarrow M\left(G \times_{\beta} B\left[\tau_{\Gamma^{\prime}}\right]\right)$ such that $\bar{\Phi} \circ \iota_{A}=\iota_{B} \circ \varphi$ and $\bar{\Phi} \circ \iota_{G, A}=\iota_{G, B}$. Moreover, using Definition 5.1, it is easy to check that $\Phi\left(G \times_{\alpha} A\left[\tau_{\Gamma}\right]\right) \subseteq G \times_{\beta} B\left[\tau_{\Gamma^{\prime}}\right]$. In the same manner, we obtain a nondegenerate pro- $C^{*}$-morphism $\Psi: G \times_{\beta} B\left[\tau_{\Gamma^{\prime}}\right] \rightarrow M\left(G \times_{\alpha} A\left[\tau_{\Gamma}\right]\right)$ such that $\bar{\Psi} \circ \iota_{B}=\iota_{A} \circ \varphi^{-1}$ and $\bar{\Psi} \circ \iota_{G, B}=\iota_{G, A}$.

From

$$
(\Phi \circ \Psi)\left(\iota_{B}(b) \iota_{G, B}(f)\right)=\Phi\left(\iota_{A} \circ \varphi^{-1}(b) \iota_{G, A}(f)\right)=\iota_{B}(b) \iota_{G, B}(f)
$$

and

$$
(\Psi \circ \Phi)\left(\iota_{A}(a) \iota_{G, A}(f)\right)=\Psi\left(\iota_{B} \circ \varphi(a) \iota_{G, B}(f)\right)=\iota_{A}(a) \iota_{G, A}(f)
$$

for all $b \in B\left[\tau_{\Gamma^{\prime}}\right], a \in A\left[\tau_{\Gamma}\right]$ and $f \in C_{c}(G)$ and Definition 5.1, we deduce that $\Phi$ and $\Psi$ are pro- $C^{*}$-isomorphisms.

Corollary 5.14. Let $\left(G, \alpha, A\left[\tau_{\Gamma}\right]\right)$ be a pro- $C^{*}$-dynamical system such that $\alpha$ is strongly bounded.

(1) Pro- $C^{*}$-algebras $G \times{ }_{\alpha} A\left[\tau_{\Gamma}\right]$ and $G \times_{\alpha} A\left[\tau_{\Gamma^{G}}\right]$ are isomorphic.

(2) $A\left[\tau_{\Gamma}\right]$ is isomorphic to a pro- $C^{*}$-subalgebra of $M\left(G \times_{\alpha} A\left[\tau_{\Gamma}\right]\right)$.

\section{THE REDUCED PRO- $C^{*}$-CROSSED PRODUCT}

Let $A\left[\tau_{\Gamma}\right]$ and $B\left[\tau_{\Gamma^{\prime}}\right]$ be two pro- $C^{*}$-algebras. For each $p_{\lambda} \in \Gamma$ and $q_{\delta} \in \Gamma^{\prime}$, the map $\vartheta_{p_{\lambda}, q_{\delta}}: A\left[\tau_{\Gamma}\right] \otimes_{\text {alg }} B\left[\tau_{\Gamma^{\prime}}\right] \rightarrow[0, \infty)$ given by

$$
\vartheta_{p_{\lambda}, q_{\delta}}(z)=\sup \left\{\|(\varphi \otimes \psi)(z)\| ; \varphi \in \mathcal{R}_{\lambda}\left(A\left[\tau_{\Gamma}\right]\right), \psi \in \mathcal{R}_{\delta}\left(B\left[\tau_{\Gamma^{\prime}}\right]\right)\right\}
$$


defines a $C^{*}$-seminorm on the algebraic tensor product $A\left[\tau_{\Gamma}\right] \otimes_{\text {alg }} B\left[\tau_{\Gamma^{\prime}}\right]$. The completion of $A\left[\tau_{\Gamma}\right] \otimes_{\text {alg }} B\left[\tau_{\Gamma^{\prime}}\right]$ with respect to the topology given by the family of $C^{*}$-seminorms $\left\{\vartheta_{p_{\lambda}, q_{\delta}} ; p_{\lambda} \in \Gamma, q_{\delta} \in \Gamma^{\prime}\right\}$ is a pro- $C^{*}$-algebra, denoted by $A\left[\tau_{\Gamma}\right] \otimes_{\min }$ $B\left[\tau_{\Gamma^{\prime}}\right]$, and called the minimal or injective tensor product of the pro- $C^{*}$-algebras $A\left[\tau_{\Gamma}\right]$ and $B\left[\tau_{\Gamma^{\prime}}\right]$ (see [F, Chapter VII]). Moreover, for each $p_{\lambda} \in \Gamma$ and $q_{\delta} \in \Gamma^{\prime}$, the $C^{*}$-algebras $\left(A\left[\tau_{\Gamma}\right] \otimes_{\min } B\left[\tau_{\Gamma^{\prime}}\right]\right)_{(\lambda, \delta)}$ and $A_{\lambda} \otimes_{\min } B_{\delta}$ are isomorphic.

Let $\left(G, \alpha, A\left[\tau_{\Gamma}\right]\right)$ be a pro- $C^{*}$-dynamical system such that $\alpha$ is strongly bounded. Since $\alpha$ is strongly bounded, for each $a \in A$, the map $t \mapsto \alpha_{t^{-1}}(a)$ defines an element in $C_{b}\left(G, A\left[\tau_{\Gamma}\right]\right)$, the pro- $C^{*}$-algebra of all bounded continuous functions from $G$ to $A\left[\tau_{\Gamma}\right]$, and so there is a map $\widetilde{\alpha}: A\left[\tau_{\Gamma}\right] \rightarrow C_{b}\left(G, A\left[\tau_{\Gamma}\right]\right)$ given by $\widetilde{\alpha}(a)(t)=\alpha_{t^{-1}}(a)$.

Lemma 6.1. Let $\left(G, \alpha, A\left[\tau_{\Gamma}\right]\right)$ be a pro- $C^{*}$-dynamical system such that $\alpha$ is strongly bounded. Then $\widetilde{\alpha}$ is a nondegenerate faithful pro- $C^{*}$-morphism from $A\left[\tau_{\Gamma}\right]$ to $M\left(A\left[\tau_{\Gamma}\right] \otimes_{\min } C_{0}(G)\right)$ with closed range. Moreover, if $\alpha$ is an inverse limit action, then $\widetilde{\alpha}$ is an inverse limit pro- $C^{*}$-morphism.

Proof. Clearly, $\widetilde{\alpha}$ is a $*$-morphism. For each $p_{\lambda} \in \Gamma$, there is $p_{\mu} \in \Gamma$ such that

$$
p_{\lambda}(a)=p_{\lambda}\left(\alpha_{e}(a)\right) \leq \sup \left\{p_{\lambda}\left(\alpha_{t}(a)\right) ; t \in G\right\}=p_{\lambda, C_{b}\left(G, A\left[\tau_{\Gamma}\right]\right)}(\widetilde{\alpha}(a)) \leq p_{\mu}(a)
$$

for all $a \in A$. Therefore, $\widetilde{\alpha}$ is an injective pro- $C^{*}$-morphism with closed range. By $\left[\mathrm{J} 2\right.$, p. 76], $C_{b}\left(G, A\left[\tau_{\Gamma}\right]\right)$ can be identified to a pro- $C^{*}$-subalgebra of $M\left(A\left[\tau_{\Gamma}\right] \otimes_{\min }\right.$ $\left.C_{0}(G)\right)$, and then $\widetilde{\alpha}$ can be regarded as a pro- $C^{*}$-morphism from $A\left[\tau_{\Gamma}\right]$ to $M\left(A\left[\tau_{\Gamma}\right]\right.$ $\left.\otimes_{\min } C_{0}(G)\right)$.

To show that $\widetilde{\alpha}$ is nondegenerate, let $\left\{e_{i}\right\}_{i \in I}$ be an approximate unit for $A\left[\tau_{\Gamma}\right]$. In the same manner as in [V, Proposition 5.1.5], we show that $\left\{\widetilde{\alpha}\left(e_{i}\right)\right\}_{i \in I}$ is strictly convergent. Indeed, let $a \in A, f \in C_{c}(G)$ and $p_{\lambda} \in \Gamma$. Then

$$
\begin{aligned}
& p_{\lambda, C_{b}\left(G, A\left[\tau_{\Gamma}\right]\right)}\left(\widetilde{\alpha}\left(e_{i}\right)(a \otimes f)-a \otimes f\right) \\
= & \sup \left\{p_{\lambda}\left(\alpha_{t^{-1}}\left(e_{i}\right) a f(t)-a f(t)\right) ; t \in G\right\} \\
\leq & \|f\|_{\infty} \sup \left\{p_{\lambda}\left(\alpha_{t^{-1}}\left(e_{i} \alpha_{t}(a)-\alpha_{t}(a)\right)\right) ; t \in \operatorname{supp}(f)\right\} \\
\leq & \|f\|_{\infty} \sup \left\{p_{\mu}\left(e_{i} \alpha_{t}(a)-\alpha_{t}(a)\right) ; t \in \operatorname{supp}(f)\right\}
\end{aligned}
$$

for some $p_{\mu} \in \Gamma$. For each $i \in I$, consider the function $f_{i}: G \rightarrow \mathbb{C}, f_{i}(t)=$ $p_{\mu}\left(e_{i} \alpha_{t}(a)-\alpha_{t}(a)\right)$. Clearly, $\left\{f_{i}\right\}_{i \in I}$ is a net of continuous functions on $G$ which is uniformly bounded and equicontinuous. Then, by Arzelà-Ascoli's theorem, it is uniformly convergent on compact subsets of $G$. Therefore, $\left\{\widetilde{\alpha}\left(e_{i}\right)\right\}_{i \in I}$ is strictly convergent, and so the pro- $C^{*}$-morphism $\widetilde{\alpha}$ is nondegenerate.

Suppose that $\alpha_{t}=\lim _{\leftarrow \lambda} \alpha_{t}^{\lambda}$ for each $t \in G$. Then $\left(\widetilde{\alpha^{\lambda}}\right)_{\lambda}$ is an inverse system of $C^{*}$-morphisms and $\widetilde{\alpha}=\lim _{\leftarrow \lambda} \widetilde{\alpha^{\lambda}}$.

Let $\varphi: A\left[\tau_{\Gamma}\right] \rightarrow M\left(B\left[\tau_{\Gamma^{\prime}}\right]\right)$ be a nondegenerate pro- $C^{*}$-morphism and let $M$ : $C_{0}(G) \rightarrow L\left(L^{2}(G)\right)$ be the representation by multiplication operators. Then there is a nondegenerate pro- $C^{*}$-morphism $\varphi \otimes M: A\left[\tau_{\Gamma}\right] \otimes_{\min } C_{0}(G) \rightarrow M\left(B\left[\tau_{\Gamma^{\prime}}\right] \otimes_{\min }\right.$ $\mathcal{K}\left(L^{2}(G)\right)$ such that $(\varphi \otimes M)(a \otimes f)=\varphi(a) \otimes M_{f}$, were $\mathcal{K}\left(L^{2}(G)\right)$ denotes the $C^{*}$-algebra of all compact operators on the Hilbert space $L^{2}(G)$. Since $\widetilde{\alpha}$ is a nondegenerate pro- $C^{*}$-morphism from $A\left[\tau_{\Gamma}\right]$ to $M\left(A\left[\tau_{\Gamma}\right] \otimes_{\min } C_{0}(G)\right), \widetilde{\varphi}=$ 
$\bar{\varphi} \otimes M \circ \widetilde{\alpha}$ is a nondegenerate pro- $C^{*}$-morphism from $A\left[\tau_{\Gamma}\right]$ to $M\left(B\left[\tau_{\Gamma^{\prime}}\right] \otimes_{\min }\right.$ $\mathcal{K}\left(L^{2}(G)\right)$.

Let $\lambda_{G}: G \rightarrow \mathcal{U}\left(L^{2}(G)\right)$ be the left representation of $G$ on $L^{2}(G)$ given by $\left(\lambda_{G}\right)_{t}(\xi)(s)=\xi\left(t^{-1} s\right)$. Then $1 \otimes \lambda_{G}: G \rightarrow \mathcal{U}\left(M\left(B\left[\tau_{\Gamma^{\prime}}\right] \otimes \mathcal{K}\left(L^{2}(G)\right)\right)\right.$, where $\left(1 \otimes \lambda_{G}\right)_{t}(b \otimes \xi)(s)=b \xi\left(t^{-1} s\right)$, is a strict continuous group morphism from $G$ to $\mathcal{U}\left(M\left(B\left[\tau_{\Gamma^{\prime}}\right] \otimes_{\min } \mathcal{K}\left(L^{2}(G)\right)\right)\right.$, and $\left(\widetilde{\varphi}, 1 \otimes \lambda_{G}\right)$ is a nondegenerate covariant morphism of $\left(G, \alpha, A\left[\tau_{\Gamma}\right]\right)$ to $B\left[\tau_{\Gamma^{\prime}}\right] \otimes_{\min } \mathcal{K}\left(L^{2}(G)\right.$. By Proposition 5.3, there is a unique nondegenerate pro- $C^{*}$-morphism $\widetilde{\varphi} \times\left(1 \otimes \lambda_{G}\right): G \times_{\alpha} A\left[\tau_{\Gamma}\right] \rightarrow$ $M\left(B\left[\tau_{\Gamma^{\prime}}\right] \otimes_{\min } \mathcal{K}\left(L^{2}(G)\right)\right.$ such that $\widetilde{\varphi} \times\left(1 \otimes \lambda_{G}\right) \circ \iota_{A}=\widetilde{\varphi}$ and $\widetilde{\varphi} \times\left(1 \otimes \lambda_{G}\right) \circ \iota_{G}=$ $1 \otimes \lambda_{G}$.

If $\varphi=\mathrm{id}_{A}$, the nondegenerate pro- $C^{*}$-morphism $\widetilde{\mathrm{id}}_{A} \times\left(1 \otimes \lambda_{G}\right): G \times{ }_{\alpha} A\left[\tau_{\Gamma}\right] \rightarrow$ $M\left(A\left[\tau_{\Gamma}\right] \otimes_{\min } \mathcal{K}\left(L^{2}(G)\right)\right.$ is denoted by $\Lambda_{A}^{G}$. It is easy to check that $\widetilde{\varphi} \times\left(1 \otimes \lambda_{G}\right)=$ $\overline{\varphi \otimes \operatorname{id}_{\mathcal{K}\left(L^{2}(G)\right)}} \circ \Lambda_{A}^{G}$.

If $\alpha$ is an inverse limit action, $\alpha_{t}=\lim _{\leftarrow \lambda} \alpha_{t}^{\lambda}$ for each $t \in G$, then it is easy to check that $\Lambda_{A}^{G}$ is an inverse limit pro- $C^{*}$-morphism, $\Lambda_{A}^{G}=\underset{\leftarrow \lambda}{\lim } \Lambda_{A_{\lambda}}^{G}$.

Definition 6.2. The reduced pro- $C^{*}$-crossed product of $A\left[\tau_{\Gamma}\right]$ by $\alpha$ is the pro$C^{*}$-subalgebra $G \times_{\alpha, r} A\left[\tau_{\Gamma}\right]$ of $M\left(A\left[\tau_{\Gamma}\right] \otimes_{\text {min }} \mathcal{K}\left(L^{2}(G)\right)\right)$ generated by the range of $\Lambda_{A}^{G}$.

Remark 6.3. From

$$
\Lambda_{A}^{G}\left(\iota_{A}(a) \iota_{G}(f)\right)=\left(\overline{\mathrm{id}_{A} \otimes M} \circ \widetilde{\alpha}\right)(a)\left(1 \otimes \lambda_{G}\right)(f)=\widetilde{\alpha}(a)\left(1 \otimes \lambda_{G}(f)\right)
$$

for all $a \in A$ and for all $f \in C_{c}(G)$, and taking into account that $G \times{ }_{\alpha}$ $A\left[\tau_{\Gamma}\right]$ is generated by $\left\{\iota_{A}(a) \iota_{G}(f) ; a \in A, f \in C_{c}(G)\right\}$, we conclude that $G \times_{\alpha, r} A\left[\tau_{\Gamma}\right]$ is the pro- $C^{*}$-subalgebra of $M\left(A\left[\tau_{\Gamma}\right] \otimes_{\min } \mathcal{K}\left(L^{2}(G)\right)\right)$ generated by $\left\{\widetilde{\alpha}(a)\left(1 \otimes \lambda_{G}(f)\right) ; a \in A, f \in C_{c}(G)\right\}$.

Remark 6.4. If $\alpha$ is an inverse limit action, $\alpha_{t}=\lim _{\leftarrow \lambda} \alpha_{t}^{\lambda}$ for each $t \in G$, then

$$
G \times_{\alpha, r} A\left[\tau_{\Gamma}\right]=\overline{\Lambda_{A}^{G}\left(G \times_{\alpha} A\left[\tau_{\Gamma}\right]\right)}=\lim _{\leftarrow \lambda} \overline{\Lambda_{A_{\lambda}}^{G}\left(G \times_{\alpha^{\lambda}} A_{\lambda}\right)}=\lim _{\leftarrow \lambda} G \times_{\alpha^{\lambda}, r} A_{\lambda}
$$

and moreover, for each $p_{\lambda} \in \Gamma$, the $C^{*}$-algebras $\left(G \times_{\alpha, r} A\left[\tau_{\Gamma}\right]\right)_{\lambda}$ and $G \times_{\alpha^{\lambda}, r} A_{\lambda}$ are isomorphic.

Remark 6.5. Since the trivial action of a locally compact group $G$ on a pro- $C^{*}-$ algebra $A\left[\tau_{\Gamma}\right]$ is an inverse limit action, the reduced pro- $C^{*}$-crossed product of $A\left[\tau_{\Gamma}\right]$ by the trivial action is the inverse limit of the reduced crossed products of $A_{\lambda}$ by the trivial action, and so it is isomorphic to the pro- $C^{*}$-algebra $A\left[\tau_{\Gamma}\right] \otimes_{\min }$ $C_{r}^{*}(G)$, where $C_{r}^{*}(G)$ is the reduced group $C^{*}$-algebra of $G$.

Proposition 6.6. Let $\left(G, \alpha, A\left[\tau_{\Gamma}\right]\right)$ and $\left(G, \beta, B\left[\tau_{\Gamma^{\prime}}\right]\right)$ be two pro- $C^{*}$-dynamical systems such that $\alpha$ and $\beta$ are strongly bounded. If $\left(G, \alpha, A\left[\tau_{\Gamma}\right]\right)$ and $\left(G, \beta, B\left[\tau_{\Gamma^{\prime}}\right]\right)$ are conjugate, then the reduced pro- $C^{*}$-crossed products associated to these pro$C^{*}$-dynamical systems are isomorphic.

Proof. Let $\varphi: A\left[\tau_{\Gamma}\right] \rightarrow B\left[\tau_{\Gamma^{\prime}}\right]$ be a pro- $C^{*}$-isomorphism such that $\varphi \circ \alpha_{t}=\beta_{t} \circ \varphi$

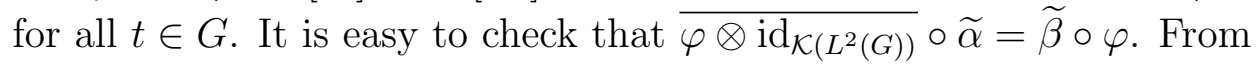

$$
\overline{\varphi \otimes \operatorname{id}_{\mathcal{K}\left(L^{2}(G)\right)}}\left(\widetilde{\alpha}(a)\left(1 \otimes \lambda_{G}(f)\right)\right)=\widetilde{\beta}(\varphi(a))\left(1 \otimes \lambda_{G}(f)\right)
$$


for all $a \in A$ and for all $f \in C_{c}(G)$, and taking into account that

$$
\overline{\operatorname{span}\left\{\widetilde{\alpha}(a)\left(1 \otimes \lambda_{G}(f)\right) ; a \in A, f \in C_{c}(G)\right\}}=G \times_{\alpha, r} A\left[\tau_{\Gamma}\right]
$$

and

$$
\overline{\operatorname{span}\left\{\widetilde{\beta}(a)\left(1 \otimes \lambda_{G}(f)\right) ; a \in B, f \in C_{c}(G)\right\}}=G \times_{\beta, r} B\left[\tau_{\Gamma^{\prime}}\right],
$$

we conclude that $\Phi_{1}=\left.\overline{\varphi \otimes \operatorname{id}_{\mathcal{K}\left(L^{2}(G)\right)}}\right|_{G \times_{\alpha, r} A\left[\tau_{\Gamma}\right]}$ is a pro- $C^{*}$-morphism from $G \times_{\alpha, r}$ $A\left[\tau_{\Gamma}\right]$ to $G \times{ }_{\beta, r} B\left[\tau_{\Gamma^{\prime}}\right]$.

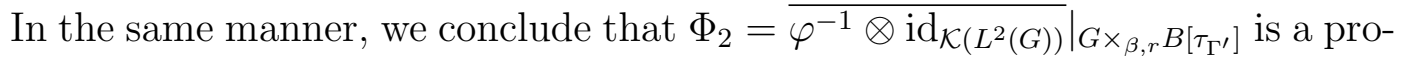
$C^{*}$-morphism from $G \times_{\beta, r} B\left[\tau_{\Gamma^{\prime}}\right]$ to $G \times_{\alpha, r} A\left[\tau_{\Gamma}\right]$. Moreover, $\Phi_{1} \circ \Phi_{2}=\operatorname{id}_{G \times \beta, r} B\left[\tau_{\Gamma^{\prime}}\right]$ and $\Phi_{2} \circ \Phi_{1}=\operatorname{id}_{G \times_{\alpha, r} A\left[\tau_{\Gamma}\right]}$, since

$$
\Phi_{1} \circ \Phi_{2}\left(\widetilde{\beta}(b)\left(1 \otimes \lambda_{G}(f)\right)\right)=\widetilde{\beta}(b)\left(1 \otimes \lambda_{G}(f)\right)
$$

for all $b \in B$ and for all $f \in C_{c}(G)$ and

$$
\Phi_{2} \circ \Phi_{1}\left(\widetilde{\alpha}(a)\left(1 \otimes \lambda_{G}(f)\right)\right)=\widetilde{\alpha}(a)\left(1 \otimes \lambda_{G}(f)\right)
$$

for all $a \in A$ and for all $f \in C_{c}(G)$. Therefore, the pro- $C^{*}$-algebras $G \times_{\alpha, r} A\left[\tau_{\Gamma}\right]$ and $G \times_{\beta, r} B\left[\tau_{\Gamma^{\prime}}\right]$ are isomorphic.

Corollary 6.7. Let $\left(G, \alpha, A\left[\tau_{\Gamma}\right]\right)$ be a pro- $C^{*}$-dynamical system such that $\alpha$ is strongly bounded. Then the pro-C ${ }^{*}$-algebras $G \times_{\alpha, r} A\left[\tau_{\Gamma}\right]$ and $G \times_{\alpha, r} A\left[\tau_{\Gamma^{G}}\right]$ are isomorphic.

Remark 6.8. If $\alpha$ is an action of an amenable locally compact group $G$ on a $C^{*}$ algebra $A$, then the $C^{*}$-morphism $\Lambda_{A}^{G}$ is injective and the full crossed product $A$ by $\alpha$ is isomorphic to the reduced crossed product of $A$ by $\alpha$. If $\alpha$ is an inverse limit action of $G$ on a pro- $C^{*}$-algebra $A\left[\tau_{\Gamma}\right]$ and $G$ is amenable, then $\Lambda_{A}^{G}$ $=\lim _{\leftarrow \lambda} \Lambda_{A_{\lambda}}^{G}$, and so $\Lambda_{A}^{G}$ is an injective pro- $C^{*}$-morphism with closed range and its inverse is continuous. Therefore, if $G$ is amenable and $\alpha$ is an inverse limit action, then the full pro- $C^{*}$-crossed product of $A\left[\tau_{\Gamma}\right]$ by $\alpha$ is isomorphic to the reduced pro- $C^{*}$-crossed product of $A\left[\tau_{\Gamma}\right]$ by $\alpha$.

Proposition 6.9. Let $\left(G, \alpha, A\left[\tau_{\Gamma}\right]\right)$ be a pro- $C^{*}$-dynamical system such that $\alpha$ is strongly bounded. If $G$ is amenable then the full pro- $C^{*}$-crossed product of $A\left[\tau_{\Gamma}\right]$ by $\alpha$ is isomorphic to the reduced pro- $C^{*}$-crossed product of $A\left[\tau_{\Gamma}\right]$ by $\alpha$.

Proof. It follows from Corollaries 5.14 and 6.7, and Remark 6.8.

\section{Pro- $C^{*}$-CROSSED Products AND TEnsor PRODUCtS}

Let $A\left[\tau_{\Gamma}\right]$ and $B\left[\tau_{\Gamma^{\prime}}\right]$ be two pro- $C^{*}$-algebras. For each $p_{\lambda} \in \Gamma$ and $q_{\delta} \in \Gamma^{\prime}$, the map $t_{p_{\lambda}, q_{\delta}}: A\left[\tau_{\Gamma}\right] \otimes_{\text {alg }} B\left[\tau_{\Gamma^{\prime}}\right] \rightarrow[0, \infty)$ given by

$$
t_{p_{\lambda}, q_{\delta}}(z)=\sup \left\{\left\|\varphi \circ \pi_{p_{\lambda}, q_{\delta}}(z)\right\| ; \varphi \text { is a } * \text {-representation of } A_{\lambda} \otimes_{\text {alg }} B_{\delta}\right\},
$$

where $\pi_{p_{\lambda}, q_{\delta}}(a \otimes b)=\pi_{\lambda}^{A}(a) \otimes \pi_{\delta}^{B}(b)$, defines a $C^{*}$-seminorm on the algebraic tensor product $A\left[\tau_{\Gamma}\right] \otimes_{\mathrm{alg}} B\left[\tau_{\Gamma^{\prime}}\right]$. The completion of $A\left[\tau_{\Gamma}\right] \otimes_{\mathrm{alg}} B\left[\tau_{\Gamma^{\prime}}\right]$ with respect to the topology given by the family of $C^{*}$-seminorms $\left\{t_{p_{\lambda}, q_{\delta}} ; p_{\lambda} \in \Gamma, q_{\delta} \in \Gamma^{\prime}\right\}$ is a pro$C^{*}$-algebra, denoted by $A\left[\tau_{\Gamma}\right] \otimes_{\max } B\left[\tau_{\Gamma^{\prime}}\right]$, and called the maximal or projective tensor product of the pro- $C^{*}$-algebras $A\left[\tau_{\Gamma}\right]$ and $B\left[\tau_{\Gamma^{\prime}}\right]$ (see [F, Chapter VII]). 
Moreover, for each $p_{\lambda} \in \Gamma$ and $q_{\delta} \in \Gamma^{\prime}$, the $C^{*}$-algebras $\left(A\left[\tau_{\Gamma}\right] \otimes_{\max } B\left[\tau_{\Gamma^{\prime}}\right]\right)_{(\lambda, \delta)}$ and $A_{\lambda} \otimes_{\max } B_{\delta}$ are isomorphic.

Remark 7.1. The trivial action of $G$ on $A\left[\tau_{\Gamma}\right]$ is an inverse limit action, and so the full pro- $C^{*}$-crossed product of $A\left[\tau_{\Gamma}\right]$ by the trivial action is isomorphic to $A\left[\tau_{\Gamma}\right] \otimes_{\max } C^{*}(G)$, where $C^{*}(G)$ is the group $C^{*}$-algebra of $G$, [J2, Corollary $1.3 .9]$.

Let $\left(G, \alpha, A\left[\tau_{\Gamma}\right]\right)$ be a pro- $C^{*}$-dynamical system such that $\alpha$ is strongly bounded and let $B\left[\tau_{\Gamma^{\prime}}\right]$ be a pro- $C^{*}$-algebra. Then $t \mapsto(\alpha \otimes \mathrm{id})_{t}$, where $(\alpha \otimes \mathrm{id})_{t}(a \otimes b)=$ $\alpha_{t}(a) \otimes b$, is a strong bounded action of $G$ on $A\left[\tau_{\Gamma}\right] \otimes_{\max } B\left[\tau_{\Gamma^{\prime}}\right]$.

The following theorem gives an "associativity" between $\times_{\alpha}$ and $\otimes_{\max }$.

Theorem 7.2. Let $\left(G, \alpha, A\left[\tau_{\Gamma}\right]\right)$ be a pro- $C^{*}$-dynamical system such that $\alpha$ is strongly bounded and let $B\left[\tau_{\Gamma^{\prime}}\right]$ be a pro- $C^{*}$-algebra. Then the pro- $C^{*}$-algebras $G \times_{\alpha \otimes i d}\left(A\left[\tau_{\Gamma}\right] \otimes_{\max } B\left[\tau_{\Gamma^{\prime}}\right]\right)$ and $\left(G \times_{\alpha} A\left[\tau_{\Gamma}\right]\right) \otimes_{\max } B\left[\tau_{\Gamma^{\prime}}\right]$ are isomorphic.

Proof. Let $\rho_{G \times \times_{\alpha} A\left[\tau_{\Gamma}\right]}: G \times_{\alpha} A\left[\tau_{\Gamma}\right] \rightarrow M\left(\left(G \times_{\alpha} A\left[\tau_{\Gamma}\right]\right) \otimes_{\max } B\left[\tau_{\Gamma^{\prime}}\right]\right)$ and $\rho_{B}:$ $B\left[\tau_{\Gamma^{\prime}}\right] \rightarrow M\left(\left(G \times{ }_{\alpha} A\left[\tau_{\Gamma}\right]\right) \otimes_{\max } B\left[\tau_{\Gamma^{\prime}}\right]\right)$ be the canonical maps. Then $\overline{\rho_{G \times \alpha_{\alpha} A\left[\tau_{\Gamma}\right]}} \circ$ $\iota_{A}: A\left[\tau_{\Gamma}\right] \rightarrow M\left(\left(G \times_{\alpha} A\left[\tau_{\Gamma}\right]\right) \otimes_{\max } B\left[\tau_{\Gamma^{\prime}}\right]\right)$ and $\rho_{B}: B\left[\tau_{\Gamma^{\prime}}\right] \rightarrow M\left(\left(G \times_{\alpha} A\left[\tau_{\Gamma}\right]\right)\right.$ $\left.\otimes_{\max } B\left[\tau_{\Gamma^{\prime}}\right]\right)$ are nondegenerate pro- $C^{*}$-morphisms with commuting ranges.

Let $j_{G \times_{\alpha \otimes \mathrm{id}} A\left[\tau_{\Gamma}\right] \otimes_{\max } B\left[\tau_{\Gamma^{\prime}}\right]}=\overline{\rho_{G \times_{\alpha} A\left[\tau_{\Gamma}\right]}} \circ \iota_{A} \otimes \rho_{B}$ and $j_{G}=\overline{\rho_{G \times_{\alpha} A\left[\tau_{\Gamma}\right]}} \circ \iota_{G}$. A simple calculus shows that $\left(j_{G \times_{\alpha \otimes \mathrm{id}} A\left[\tau_{\Gamma}\right] \otimes_{\max } B\left[\tau_{\Gamma^{\prime}}\right]}, j_{G}\right)$ is a nondegenerate covariant pro- $C^{*}$-morphism from $\left(G, \alpha \otimes \mathrm{id}, A\left[\tau_{\Gamma}\right] \otimes_{\max } B\left[\tau_{\Gamma^{\prime}}\right]\right)$ to $M\left(\left(G \times{ }_{\alpha} A\left[\tau_{\Gamma}\right]\right) \otimes_{\max }\right.$ $\left.B\left[\tau_{\Gamma^{\prime}}\right]\right)$. Moreover, from

$$
\begin{aligned}
& j_{G \times_{\alpha \otimes \mathrm{id}} A\left[\tau_{\Gamma}\right] \otimes_{\max } B\left[\tau_{\Gamma^{\prime}}\right]}(a \otimes b) j_{G}(f) \\
= & \overline{\rho_{G \times_{\alpha} A\left[\tau_{\Gamma}\right]}}\left(\iota_{A}(a)\right) \rho_{B}(b) \overline{\rho_{G \times_{\alpha}} A\left[\tau_{\Gamma}\right]}\left(\iota_{G}(f)\right) \\
= & \overline{\rho_{G \times_{\alpha} A\left[\tau_{\Gamma}\right]}}\left(\iota_{A}(a)\right) \overline{\rho_{G \times_{\alpha} A\left[\tau_{\Gamma}\right]}}\left(\iota_{G}(f)\right) \rho_{B}(b) \\
= & \rho_{G \times_{\alpha} A\left[\tau_{\Gamma}\right]}\left(\iota_{A}(a) \iota_{G}(f)\right) \rho_{B}(b)
\end{aligned}
$$

for all $a \in A$, for all $b \in B$ and for all $f \in C_{c}(G)$, and taking into account that $\left\{\iota_{A}(a) \iota_{G}(f) ; a \in A, f \in C_{c}(G)\right\}$ generates $G \times_{\alpha} A\left[\tau_{\Gamma}\right]$ and $\left\{\rho_{G \times_{\alpha} A\left[\tau_{\Gamma}\right]}(z) \rho_{B}(b)\right.$; $\left.z \in G \times{ }_{\alpha} A\left[\tau_{\Gamma}\right], b \in B\right\}$ generates $\left(G \times{ }_{\alpha} A\left[\tau_{\Gamma}\right]\right) \otimes_{\max } B\left[\tau_{\Gamma^{\prime}}\right]$, we conclude that

$$
\begin{aligned}
& \overline{\operatorname{span}\left\{j_{G \times_{\alpha \otimes \mathrm{id}} A\left[\tau_{\Gamma}\right] \otimes_{\max } B\left[\tau_{\Gamma^{\prime}}\right]}(a \otimes b) j_{G}(f) ; a \in A, b \in b, f \in C_{c}(G)\right\}} \\
= & \left(G \times_{\alpha} A\left[\tau_{\Gamma}\right]\right) \otimes_{\max } B\left[\tau_{\Gamma^{\prime}}\right] .
\end{aligned}
$$

Let $(\varphi, u, \mathcal{H})$ be a nondegenerate covariant representation of $\left(G, \alpha \otimes \mathrm{id}, A\left[\tau_{\Gamma}\right] \otimes_{\max }\right.$ $\left.B\left[\tau_{\Gamma^{\prime}}\right]\right)$. Then $(\varphi, \mathcal{H})$ is a nondegenerate representation of $A\left[\tau_{\Gamma}\right] \otimes_{\max } B\left[\tau_{\Gamma^{\prime}}\right]$, and so there is a nondegenerate representation $\left(\varphi_{(\lambda, \delta)}, \mathcal{H}\right)$ of $A_{\lambda} \otimes_{\max } B_{\delta}$ such that $\varphi_{(\lambda, \delta)} \circ \pi_{(\lambda, \delta)}^{A\left[\tau_{\Gamma}\right] \otimes_{\max } B\left[\tau_{\Gamma^{\prime}}\right]}=\varphi$. Let $\left(\varphi_{\lambda}, \mathcal{H}\right)$ and $\left(\varphi_{\delta}, \mathcal{H}\right)$ be the nondegenerate representations of $A_{\lambda}$, respectively $B_{\delta}$ with commuting ranges such that $\varphi_{(\lambda, \delta)}(a \otimes b)=$ $\varphi_{\lambda}(a) \varphi_{\delta}(b)$ for all $a \in A_{\lambda}$ and $b \in B_{\delta}$. Then $\left(\varphi_{\lambda} \circ \pi_{\lambda}^{A}, u, \mathcal{H}\right)$ is a nondegenerate covariant representation of $\left(G, \alpha, A\left[\tau_{\Gamma}\right]\right)$, and so there is a nondegenerate representation $\left(\Phi_{1}, \mathcal{H}\right)$ of $G \times_{\alpha} A\left[\tau_{\Gamma}\right]$ such that $\overline{\Phi_{1}} \circ \iota_{A}=\varphi_{\lambda} \circ \pi_{\lambda}^{A}$ and $\overline{\Phi_{1}} \circ \iota_{G}=u$. It is easy to check that $\left(\Phi_{1}, \mathcal{H}\right)$ and $\left(\Phi_{2}, \mathcal{H}\right)$, where $\Phi_{2}=\varphi_{\delta} \circ \pi_{\delta}^{B}$, are nondegenerate representations of $G \times{ }_{\alpha} A\left[\tau_{\Gamma}\right]$ respectively $B\left[\tau_{\Gamma^{\prime}}\right]$ with commuting ranges. Let 
$(\Phi, \mathcal{H})$ be the nondegenerate representation of $\left(G \times_{\alpha} A\left[\tau_{\Gamma}\right]\right) \otimes_{\max } B\left[\tau_{\Gamma^{\prime}}\right]$ given by $\Phi(z \otimes b)=\Phi_{1}(z) \Phi_{2}(b)$. Then

$$
\begin{aligned}
& \bar{\Phi}\left(j_{G \times_{\alpha \otimes \mathrm{id}} A\left[\tau_{\Gamma}\right] \otimes_{\max } B\left[\tau_{\Gamma^{\prime}}\right]}(a \otimes b)\right) \\
= & \bar{\Phi}\left(\overline{\rho_{G \times_{\alpha} A\left[\tau_{\Gamma}\right]}}\left(\iota_{A}(a)\right) \rho_{B}(b)\right)=\overline{\Phi_{1}}\left(\left(\iota_{A}(a)\right) \overline{\Phi_{2}}\left(\rho_{B}(b)\right)\right. \\
= & \left(\varphi_{\lambda} \circ \pi_{\lambda}^{A}(a)\right)\left(\varphi_{\delta} \circ \pi_{\delta}^{B}(b)\right)=\varphi_{(\lambda, \delta)}\left(\pi_{\lambda}^{A}(a) \otimes \pi_{\delta}^{B}(b)\right) \\
= & \varphi_{(\lambda, \delta)} \circ \pi_{(\lambda, \delta)}^{A\left[\tau_{\Gamma}\right] \otimes_{\max } B\left[\tau_{\Gamma^{\prime}}\right]}(a \otimes b)=\varphi(a \otimes b)
\end{aligned}
$$

for all $a \in A$ and $b \in B$, and

$$
\bar{\Phi}\left(j_{G}(f)\right)=\bar{\Phi}\left(\overline{\rho_{G \times \alpha} A\left[\tau_{\Gamma}\right]} \circ \iota_{G}(f)\right)=\overline{\Phi_{1}}\left(\iota_{G}(f)\right)=u(f)
$$

for all $f \in C_{c}(G)$. Therefore, by Definition 5.1 and Corollary 5.4, the pro- $C^{*}$ algebras $G \times_{\alpha \otimes \text { id }} A\left[\tau_{\Gamma}\right] \otimes_{\max } B\left[\tau_{\Gamma^{\prime}}\right]$ and $\left(G \times_{\alpha} A\left[\tau_{\Gamma}\right]\right) \otimes_{\max } B\left[\tau_{\Gamma^{\prime}}\right]$ are isomorphic.

Let $\left(G, \alpha, A\left[\tau_{\Gamma}\right]\right)$ be a pro- $C^{*}$-dynamical system such that $\alpha$ is strongly bounded and let $B\left[\tau_{\Gamma^{\prime}}\right]$ be a pro- $C^{*}$-algebra. Then $t \mapsto(\alpha \otimes \mathrm{id})_{t}$, where $(\alpha \otimes \mathrm{id})_{t}(a \otimes b)=$ $\alpha_{t}(a) \otimes b$, is a strong bounded action of $G$ on $A\left[\tau_{\Gamma}\right] \otimes_{\min } B\left[\tau_{\Gamma^{\prime}}\right]$.

The following theorem gives an "associativity" between $\times_{\alpha, r}$ and $\otimes_{\min }$.

Theorem 7.3. Let $\left(G, \alpha, A\left[\tau_{\Gamma}\right]\right)$ be a pro- $C^{*}$-dynamical system such that $\alpha$ is strongly bounded and let $B\left[\tau_{\Gamma^{\prime}}\right]$ be a pro- $C^{*}$-algebra. Then the pro- $C^{*}$-algebras $G \times_{\alpha \otimes i d, r}\left(A\left[\tau_{\Gamma}\right] \otimes_{\min } B\left[\tau_{\Gamma^{\prime}}\right]\right)$ and $\left(G \times_{\alpha, r} A\left[\tau_{\Gamma}\right]\right) \otimes_{\min } B\left[\tau_{\Gamma^{\prime}}\right]$ are isomorphic.

Proof. The map id ${ }_{A} \otimes \sigma_{B, \mathcal{K}\left(L^{2}(G)\right)}: A\left[\tau_{\Gamma}\right] \otimes_{\min } B\left[\tau_{\Gamma^{\prime}}\right] \otimes_{\min } \mathcal{K}\left(L^{2}(G)\right) \rightarrow A\left[\tau_{\Gamma}\right] \otimes_{\min }$ $\mathcal{K}\left(L^{2}(G)\right) \otimes_{\min } B\left[\tau_{\Gamma^{\prime}}\right]$ given by

$$
\operatorname{id}_{A} \otimes \sigma_{B, \mathcal{K}\left(L^{2}(G)\right)}(a \otimes b \otimes T)=a \otimes T \otimes b
$$

is a pro- $C^{*}$-isomorphism. Moreover, $\mathrm{id}_{A} \otimes \sigma_{B, \mathcal{K}\left(L^{2}(G)\right)}$ is an inverse limit of $C^{*}$ isomorphisms. From

$$
\overline{\operatorname{id}_{A} \otimes \sigma_{B, \mathcal{K}\left(L^{2}(G)\right)}}\left(\widetilde{\alpha \otimes \mathrm{id}}(a \otimes b)\left(1 \otimes \lambda_{G}(f)\right)=\widetilde{\alpha}(a)\left(1_{M\left(A\left[\tau_{\Gamma}\right]\right)} \otimes \lambda_{G}(f) \otimes b\right)\right.
$$

for all $a \in A$, for all $b \in B$ and for all $f \in C_{c}(G)$, and Remark 6.3, we deduce that

$$
\left.\overline{\operatorname{id}_{A} \otimes \sigma_{B, \mathcal{K}\left(L^{2}(G)\right)}}\right|_{G \times_{\alpha \otimes \mathrm{id}, r} A\left[\tau_{\Gamma}\right] \otimes_{\min } B\left[\tau_{\Gamma^{\prime}}\right]}
$$

is a pro- $C^{*}$-isomorphism from $G \times_{\alpha \otimes \mathrm{id}, r}\left(A\left[\tau_{\Gamma}\right] \otimes_{\min } B\left[\tau_{\Gamma^{\prime}}\right]\right)$ onto $\left(G \times_{\alpha, r} A\left[\tau_{\Gamma}\right]\right)$ $\otimes_{\min } B\left[\tau_{\Gamma^{\prime}}\right]$. Therefore, the pro- $C^{*}$-algebras $\left(G \times_{\alpha, r} A\left[\tau_{\Gamma}\right]\right) \otimes_{\min } B\left[\tau_{\Gamma^{\prime}}\right]$ and $G \times_{\alpha \otimes \mathrm{id}, r}$ $\left(A\left[\tau_{\Gamma}\right] \otimes_{\min } B\left[\tau_{\Gamma^{\prime}}\right]\right)$ are isomorphic.

Acknowledgement. The author was supported by the grant of the Romanian National Authority for Scientific Research, CNCS - UEFISCDI, project number PN-II-ID-PCE-2012-4-0201. 


\section{REFERENCES}

A. C. Apostol, $b^{*}$-algebras and their representation, J. London Math. Soc. (2) 3 (1971), no. 1, 30-38.

E. S. Echterhoff, Crossed products, the Mackey-Rieffel-Green machine and applications, arXiv:1006.4975v2.

F. M. Fragoulopoulou, Topological algebras with involution, North-Holland Mathematics Studies, 200. Elsevier Science B.V., Amsterdam, 2005.

I. A. Inoue, Locally $C^{*}$-algebras, Mem. Faculty Sci. Kyushu Univ. Ser. A 25 (1971), 197-235.

J1. M. Joiţa, On bounded module maps between Hilbert modules over locally $C^{*}$-algebras, Acta Math. Univ. Comenianae 74(2005), no. 1, 71-78.

J2. M. Joiţa, Crossed products of locally $C^{*}$-algebras, Editura Academiei Române, Bucharest, 2007.

J3. M. Joiţa, Crossed products of pro- $C^{*}$-algebras and Hilbert pro- $C^{*}$-modules, Bull. Malays. Math. Sci. Soc. (to appear).

J4. M. Joița, Crossed products of locally $C^{*}$-algebras, Rocky Mountain J. Math. 37(2007), no. 5, 1623-1644.

M. A. Mallios, Topological Algebras. Selected Topics, North-Holland, Amsterdam, 1986.

P1. N.C. Phillips, Inverse limits of $C^{*}$-algebras, J. Operator Theory 19(1988), no. 1, 159-195.

P2. N.C. Phillips, Representable K-theory for $\sigma$-C*-algebras, K-Theory 3(1989), no. 5, 441478.

R. I. Raeburn, On crossed products and Takai duality, Proc. Edinburgh Math. Soc. (2) 31(1988), no. 2, 321-330.

V. J.M. Vallin, $C^{*}$-algébres de Hopf et $C^{*}$-algébres de Kac, Proc. London Math. Soc.(3) 50 (1985), no. 1, 131-174.

W. D.P. Williams, Crossed products of $C^{*}$-algebras. Mathematical Surveys and Monographs, Vol 134, Amer. Math. Soc., 2007.

Department of Mathematics, Faculty of Applied Sciences, University Politehnica

of Bucharest, Spl. Independentei nr. 313, Bucharest, 060042, Romania;

Simion Stoilow Institute of Mathematics of the Roumanian Academy, 21 Calea

Grivitei Street, 010702 Bucharest, Romania.

E-mail address: mjoita@fmi.unibuc.ro

$U R L:$ http://sites.google.com/a/g.unibuc.ro/maria-joita/ 